\title{
A fully discrete, stable and conservative summation-by-parts formulation for deforming interfaces
}

Samira Nikkar and Jan Nordström

Journal Article

\section{Tweet}

N.B.: When citing this work, cite the original article.

Original Publication:

Samira Nikkar and Jan Nordström, A fully discrete, stable and conservative summation-byparts formulation for deforming interfaces, Journal of Computational Physics, 2017. 339, pp.500-524.

http://dx.doi.org/10.1016/j.jcp.2017.02.047

Copyright: Elsevier

http://www.elsevier.com/

Postprint available at: Linköping University Electronic Press

http://urn.kb.se/resolve?urn=urn:nbn:se:liu:diva-136814

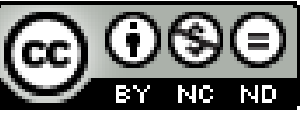




\title{
A Fully Discrete, Stable and Conservative Summation-by-parts Formulation for Deforming Interfaces
}

\author{
Samira Nikkar ${ }^{\mathrm{a}}$, Jan Nordström ${ }^{\mathrm{b}}$ \\ ${ }^{a}$ Department of Mathematics, Computational Mathematics, Linköping University, SE-581 83 \\ Linköping, Sweden (samira.nikkar@liu.se). \\ ${ }^{b}$ Department of Mathematics, Computational Mathematics, Linköping University, SE-581 83 \\ Linköping, Sweden( jan.nordstrom@liu.se).
}

\begin{abstract}
We introduce an interface/coupling procedure for hyperbolic problems posed on time-dependent curved multi-domains. First, we transform the problem from Cartesian to boundary-conforming curvilinear coordinates and apply the energy method to derive well-posed and conservative interface conditions.

Next, we discretize the problem in space and time by employing finite difference operators that satisfy a summation-by-parts rule. The interface condition is imposed weakly using a penalty formulation. We show how to formulate the penalty operators such that the coupling procedure is automatically adjusted to the movements and deformations of the interface, while both stability and conservation conditions are respected.

The developed techniques are illustrated by performing numerical experiments on the linearized Euler equations and the Maxwell equations. The results corroborate the stability and accuracy of the fully discrete approximations.

Keywords: Finite difference, High order accuracy, Deforming domains, Time-dependent interface, Well-posedness, Conservation, Summation-by-parts, Stability, Hyperbolic problems.
\end{abstract}

\section{Introduction}

Multi-block schemes and in particular interface procedures that use Summationby-Parts (SBP) operators together with the Simultaneous Approximation Term (SAT) technique [2], have previously been investigated in terms of conservation, 
stability and accuracy $[3,5,6,10,11,26,27,28,29]$. The focus of the SBP-SAT methodology has been, so far, mostly on time-independent spatial domains with a notable exception being [1].

In this article, we extend the techniques introduced in [1] for handling timedependent boundaries in a single domain problem, to a multi-domain context with deforming interfaces. The new time-dependent interface formulation is conservative, provably stable and high order accurate.

The rest of this article proceeds as follows. We start, in section 2, by transforming the continuous problem from Cartesian to curvilinear coordinates. Next, we study the problem using the energy method, our analytical tool, and derive conditions for conservation and well-posedness. Section 3 deals with the discrete problem where we study conservation and stability of the interface procedures and show the similarities with the continuous problem. In section 4, numerical experiments are performed to show the accuracy and the usefulness of the scheme. Finally, we summarize and draw conclusions in section 5.

\section{The continuous problem}

Consider one hyperbolic problem with solution $W$ posed on two nearby spatial domains, as

$$
\begin{aligned}
& U_{t}+\hat{A} U_{x}+\hat{B} U_{y}=0, \quad(x, y) \in \Omega_{L}(t), \quad t \in[0, T], \\
& V_{t}+\hat{A} V_{x}+\hat{B} V_{y}=0, \quad(x, y) \in \Omega_{R}(t), \quad t \in[0, T] .
\end{aligned}
$$

The solutions $U, V$ represent the left $L$ and right $R$ values of the continuous solution $W$ posed on the union of $\Omega_{L}(t)$ and $\Omega_{R}(t)$, where $\Omega_{L, R}(t)$ are the timedependent sub-domains. In (1), $x$ and $y$ are the spatial coordinates and $t$ represents time. The matrices $\hat{A}$ and $\hat{B}$ are constant, symmetric $[19,25]$ and of size $l \times l$. We focus on the case where the deformations of $\Omega_{L, R}(t)$ are mainly caused by the moving and/or deforming interface $I(t)$, see Figure 1 .

Next, two time-dependent invertible Lagrangian-Eulerian transformations [22] of $\Omega_{L, R}(t)$ from Cartesian to curvilinear coordinates as

$$
x(\tau, \xi, \eta) \rightleftharpoons \xi(t, x, y), \quad y(\tau, \xi, \eta) \rightleftharpoons \eta(t, x, y), \quad \tau=t,
$$

are introduced. We consider boundary-conforming curvilinear coordinates where the boundaries of $\Omega_{L, R}$ are composed of segments with constant $\xi, \eta$, resulting in fixed spatial sub-domains after the transformations [30]. The fixed sub-domains are denoted by $\Phi_{L, R}$ and shown schematically in Figure 2. The interface between $\Phi_{L}$ and $\Phi_{R}$, denoted by $I$, is now time-independent in $\eta, \xi$ space. 


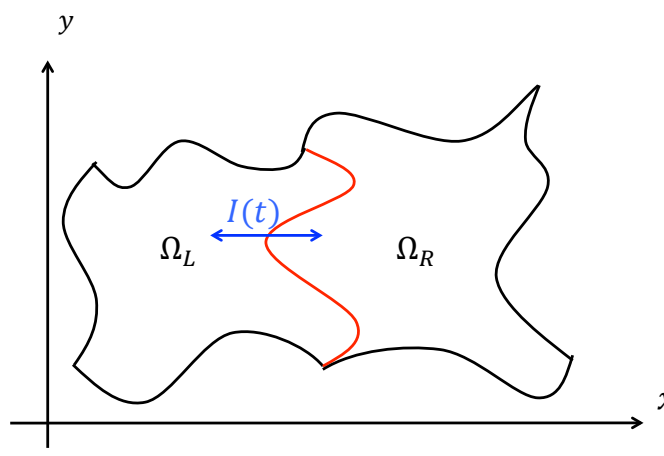

Figure 1: A schematic of the domains $\Omega_{L, R}(t)$ and the time-dependent interface $I(t)$

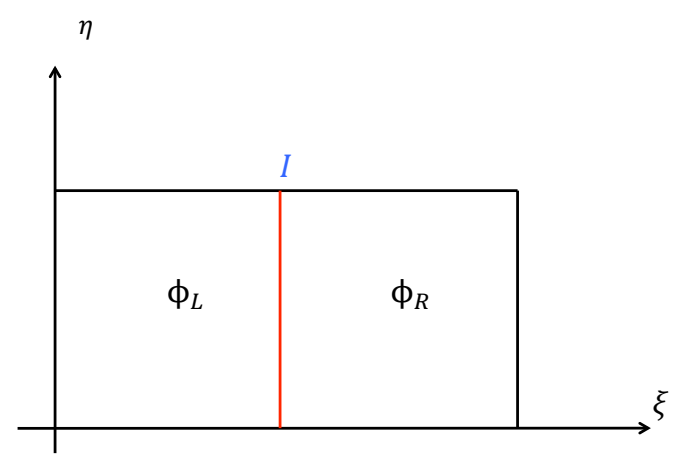

Figure 2: A schematic of the transformed domains $\Phi_{L, R}$ and the time-independent interface $I$.

The transformations we have used both satisfy

$$
\left[\begin{array}{l}
\partial / \partial \xi \\
\partial / \partial \eta \\
\partial / \partial \tau
\end{array}\right]=\underbrace{\left[\begin{array}{lll}
x_{\xi} & y_{\xi} & 0 \\
x_{\eta} & y_{\eta} & 0 \\
x_{\tau} & y_{\tau} & 1
\end{array}\right]}_{:=[J]}\left[\begin{array}{l}
\partial / \partial x \\
\partial / \partial y \\
\partial / \partial t
\end{array}\right]
$$

where the subscripts $\xi, \eta$ and $\tau$ denote partial derivatives and $[J]$ is the Jacobian matrix of the transformation. By considering the Jacobian matrix of the inverse transformation, the following metric relations are obtained [12, 30]

$$
\begin{array}{lll}
J \xi_{t}=x_{\eta} y_{\tau}-x_{\tau} y_{\eta}, & J \xi_{x}=y_{\eta}, & J \xi_{y}=-x_{\eta} \\
J \eta_{t}=y_{\xi} x_{\tau}-x_{\xi} y_{\tau}, & J \eta_{x}=-y_{\xi}, & J \eta_{y}=x_{\xi}
\end{array}
$$

where $J=x_{\xi} y_{\eta}-x_{\eta} y_{\xi}>0$ is the determinant of $[J]$.

For non-singular (invertible) transformations, the Geometric Conservation Law (GCL) $[12,30]$ holds, i.e.

$$
\begin{array}{r}
J_{\tau}+\left(J \xi_{t}\right)_{\xi}+\left(J \eta_{t}\right)_{\eta}=0 \\
\left(J \xi_{x}\right)_{\xi}+\left(J \eta_{x}\right)_{\eta}=0 \\
\left(J \xi_{y}\right)_{\xi}+\left(J \eta_{y}\right)_{\eta}=0
\end{array}
$$

Remark 1. For ease of presentation, we have not distinguished between the left and right transformations in (2), (3), (4) and (5) whereas in the remainder of the article, we will show this by using the subscripts $L$ and $R$. 
Next, the governing equations in (1) are expressed in terms of $\xi, \eta$ and $\tau$ by using the chain rule and multiplying the results with $J_{L, R}$, as

$$
\begin{aligned}
& J_{L} U_{\tau}+A_{L} U_{\xi}+B_{L} U_{\eta}=0, \quad(\xi, \eta) \in \Phi_{L}, \quad \tau \in[0, T], \\
& J_{R} V_{\tau}+A_{R} V_{\xi}+B_{R} V_{\eta}=0, \quad(\xi, \eta) \in \Phi_{R}, \quad \tau \in[0, T],
\end{aligned}
$$

where

$$
\begin{aligned}
& A_{L, R}=\left(J \xi_{t}\right)_{L, R} I+\left(J \xi_{x}\right)_{L, R} \hat{A}+\left(J \xi_{y}\right)_{L, R} \hat{B}, \\
& B_{L, R}=\left(J \eta_{t}\right)_{L, R} I+\left(J \eta_{x}\right)_{L, R} \hat{A}+\left(J \eta_{y}\right)_{L, R} \hat{B},
\end{aligned}
$$

and $I$ is the identity matrix of size $l$. The GCL in (5) applied to (7) results in

$$
\begin{aligned}
& \left(J_{L}\right)_{\tau} I+\left(A_{L}\right)_{\xi}+\left(B_{L}\right)_{\eta}=0 \\
& \left(J_{R}\right)_{\tau} I+\left(A_{R}\right)_{\xi}+\left(B_{R}\right)_{\eta}=0 .
\end{aligned}
$$

By using (8), we can re-write (6) on conservative form as

$$
\begin{aligned}
& \left(J_{L} U\right)_{\tau}+\left(A_{L} U\right)_{\xi}+\left(B_{L} U\right)_{\eta}=0, \quad(\xi, \eta) \in \Phi_{L}, \quad \tau \in[0, T] \\
& \left(J_{R} V\right)_{\tau}+\left(A_{R} V\right)_{\xi}+\left(B_{R} V\right)_{\eta}=0, \quad(\xi, \eta) \in \Phi_{R}, \quad \tau \in[0, T] .
\end{aligned}
$$

\subsection{Continuous conservation}

If we integrate (9) in space and time we can directly see that the solution $U, V$ is conserved. In a conservation law, the total quantity of a conserved variable in the region of interest changes only as a result of the flux through the boundaries of the region. In this article, however, we use a broader definition of conservation motivated by the original proof of the Lax-Wandroff theorem [32]. We demand that all moments of the flux against an arbitrary test function telescope across the domain.

We multiply the first and second equations in (9), with the transpose of a smooth vector function $\phi(\tau, \xi, \eta)$ with compact support at the spatial and temporal boundaries. Integration in space and time together with the use of the GaussGreen theorem leads to

$$
\begin{aligned}
& \int_{0}^{T} \iint_{\Phi_{L}}\left[U^{T}\left[\left(J_{L} \phi_{L}\right)_{\tau}+\left(A_{L} \phi_{L}\right)_{\xi}+\left(B_{L} \phi_{L}\right)_{\eta}\right]\right]^{T} d \Phi d \tau+ \\
& \int_{0}^{T} \iint_{\Phi_{R}}\left[V^{T}\left[\left(J_{R} \phi_{R}\right)_{\tau}+\left(A_{R} \phi_{R}\right)_{\xi}+\left(B_{R} \phi_{R}\right)_{\eta}\right]\right]^{T} d \Phi d \tau=\int_{0}^{T} \int_{I} \underbrace{\phi_{I}^{T}\left(A_{L_{I}} U_{I}-A_{R_{I}} V_{I}\right)}_{I T} d \eta d \tau .
\end{aligned}
$$

In (10), the subscript $I$ indicates restrictions to the interface and $I T$ denotes the interface term. The details of how to get (10) are given in Appendix A. 
Since $U_{I}=V_{I}=W_{I}$, demanding

$$
A_{L_{I}}=A_{R_{I}}
$$

will remove $I T$. To satisfy (11), the left and right metrics terms need to have identical values at the interface by which (10) becomes an integral statement of the original single problem and conservation is respected. This indicates that the conservation condition (11) must be satisfied also in the numerical approximations, as we will show later.

For later comparison, we split the integral arguments in (10) and obtain

$$
\begin{gathered}
\int_{0}^{T} \iint_{\Phi_{L}}\left[\frac { 1 } { 2 } U ^ { T } \left(\left[\left(J_{L} \phi_{L}\right)_{\tau}+J_{L}\left(\phi_{L}\right)_{\tau}+\left(J_{L}\right)_{\tau} \phi_{L}\right]+\left[\left(A_{L} \phi_{L}\right)_{\xi}+A_{L}\left(\phi_{L}\right)_{\xi}+\left(A_{L}\right)_{\xi} \phi_{L}\right]+\right.\right. \\
\left.\left.\left[\left(B_{L} \phi_{L}\right)_{\eta}+B_{L}\left(\phi_{L}\right)_{\eta}+\left(B_{L}\right)_{\eta} \phi_{L}\right]\right)\right]^{T} d \Phi d \tau+ \\
\int_{0}^{T} \iint_{\Phi_{R}}\left[\frac { 1 } { 2 } V ^ { T } \left(\left[\left(J_{R} \phi_{R}\right)_{\tau}+J_{R}\left(\phi_{R}\right)_{\tau}+\left(J_{R}\right)_{\tau} \phi_{R}\right]+\left[\left(A_{R} \phi_{R}\right)_{\xi}+A_{R}\left(\phi_{R}\right)_{\xi}+\left(A_{R}\right)_{\xi} \phi_{R}\right]+\right.\right. \\
\left.\left.\left[\left(B_{R} \phi_{R}\right)_{\eta}+B_{R}\left(\phi_{R}\right)_{\eta}+\left(B_{R}\right)_{\eta} \phi_{R}\right]\right)\right]^{T} d \Phi d \tau=0 .
\end{gathered}
$$

The result of this section is summarized in the following proposition.

Proposition 1. The continuous problem (9) with the condition (11) is conservative.

\subsection{Well-posedness}

The energy method (multiplying (9) with the transpose of the solution and integrating over the spatial and temporal domains) together with the use of GreenGauss theorem results in

$$
\begin{gathered}
\left.\|U(T, \xi, \eta)\|_{J_{L}}^{2}=\| U(0, \xi, \eta)\right) \|_{J_{L}}^{2}-\int_{0}^{T} \oint_{\delta \Phi_{L}} U^{T} C_{L} U d s d \tau, \\
\left.\|V(T, \xi, \eta)\|_{J_{R}}^{2}=\| V(0, \xi, \eta)\right) \|_{J_{R}}^{2}-\int_{0}^{T} \oint_{\delta \Phi_{R}} V^{T} C_{R} V d s d \tau .
\end{gathered}
$$

In (13), the norm is defined as $\|W\|_{J_{L, R}}^{2}=\iint_{\Phi_{L, R}} W^{T} J_{L, R} W d \xi d \eta$ for $W \in\{U, V\}$. Moreover, $C_{L, R}=\left(A_{L, R}, B_{L, R}\right) \cdot n$ where $n=(\alpha, \beta)$ is the outward pointing normal vector from $\Phi_{L, R}$. Further, $C_{L, R}$ can be expanded as $C_{L, R}=\alpha A_{L, R}+\beta B_{L, R}$ and due to symmetry be decomposed into $C_{L, R}=X_{L, R} \Lambda_{L, R} X_{L, R}^{T}$ where $\Lambda_{L, R}$ is the matrix of eigenvalues of $C_{L, R}$ and $X_{L, R}$ is the corresponding eigenvector matrix. 
At the far-field boundaries, we specify characteristic boundary conditions as

$$
\begin{aligned}
& \left(X_{L}^{T} U\right)_{j}=\left(X_{L}^{T} U_{\infty}\right)_{j} \text { if }\left(\Lambda_{L}\right)_{j j}<0 \\
& \left(X_{R}^{T} V\right)_{j}=\left(X_{R}^{T} V_{\infty}\right)_{j} \text { if }\left(\Lambda_{R}\right)_{j j}<0
\end{aligned}
$$

to control the energy growth. In (14), $U_{\infty}, V_{\infty}$ are external data and $j \in\{1, \ldots, l\}$.

By substituting (14) in (13) and considering the initial conditions $U(0, \xi, \eta)=$ $f_{L}$ and $V(0, \xi, \eta)=f_{R}$, we arrive at

$$
\begin{aligned}
\|U(T, \xi, \eta)\|_{J_{L}}^{2}+\|V(T, \xi, \eta)\|_{J_{R}}^{2} & +\int_{0}^{T} \int_{\delta \Phi_{L} \backslash I}\left(X_{L}^{T} U\right)^{T} \Lambda_{L}^{+}\left(X_{L}^{T} U\right) d s d \tau \\
& +\int_{0}^{T} \int_{\delta \Phi_{R} \backslash I}\left(X_{R}^{T} V\right)^{T} \Lambda_{R}^{+}\left(X_{R}^{T} V\right) d s d \tau \\
& =\left\|f_{L}\right\|_{J_{L}}^{2}-\int_{0}^{T} \int_{\delta \Phi_{L} \backslash I}\left(X_{L}^{T} U_{\infty}\right)^{T} \Lambda_{L}^{-}\left(X_{L}^{T} U_{\infty}\right) d s d \tau \\
& +\left\|f_{R}\right\|_{J_{R}}^{2}-\int_{0}^{T} \int_{\delta \Phi_{R} \backslash I}\left(X_{R}^{T} V_{\infty}\right)^{T} \Lambda_{R}^{-}\left(X_{R}^{T} V_{\infty}\right) d s d \tau, \\
& -\int_{0}^{T} \int_{I} U_{I}^{T} C_{L} U_{I}-\int_{0}^{T} \int_{I} V_{I}^{T} C_{R} V_{I},
\end{aligned}
$$

where the positive and negative superscripts in $\Lambda_{L}^{+,-}, \Lambda_{R}^{+,-}$and $\Lambda_{I}^{+,-}$restrict $\Lambda_{L, R, I}$ to the non-negative and negative eigenvalues, respectively. At the interface, we have $n_{L}=(1,0)^{T}, n_{R}=(-1,0)^{T}$ and therefore $C_{L}=A_{L_{I}}$ and $C_{R}=-A_{R_{I}}$. By considering again (11) together with $U_{I}=V_{I}$, the interface terms vanish.

The result of this section is summarized in the following proposition.

Proposition 2. The continuous problem (9) with the interface condition (11), and the boundary conditions (14) is strongly well-posed, and satisfies the bound in (15).

\section{The discrete problem}

We discretize $\Phi_{L, R}$ by constructing meshes of $N_{L, R}+1$ and $M+1$ nodes in the $\xi$ and $\eta$ directions, respectively. We consider matching grid points at the interface in the $\eta$ direction. For non-conforming grids, interpolation techniques $[6,24]$ must be used in order to couple the blocks, due to the added difficulty 
of a moving interface we refrain from this technical complication in this article. We use $K+1$ time levels from 0 to $T$ and tensor products to arrange the fully discrete numerical solutions. As an example, the numerical solution on the left sub-domain is organized as

$$
\mathbf{U}=\left[\begin{array}{c}
\mathbf{U}_{0} \\
\vdots \\
{\left[\mathbf{U}_{k}\right]} \\
\vdots \\
\mathbf{U}_{K}
\end{array}\right] ;\left[\mathbf{U}_{k}\right]=\left[\begin{array}{c}
\mathbf{U}_{0} \\
\vdots \\
{\left[\mathbf{U}_{i}\right]} \\
\vdots \\
\mathbf{U}_{N_{L}}
\end{array}\right]_{k} ;\left[\mathbf{U}_{i}\right]_{k}=\left[\begin{array}{c}
\mathbf{U}_{0} \\
\vdots \\
\mathbf{U}_{j} \\
\vdots \\
\mathbf{U}_{M}
\end{array}\right]_{k i}
$$

in which $\mathbf{U}_{k i j} \approx U\left(\tau_{k}, \xi_{i}, \eta_{j}\right)$, where $\left(\xi_{i}, \eta_{j}\right) \in \Phi_{L}$. The fully discrete numerical solution corresponding to the right sub-domain is denoted by $\mathbf{V}$ and arranged in the same way. In Figure 3, a schematic of the mesh is shown to clarify the indexing used in (16).

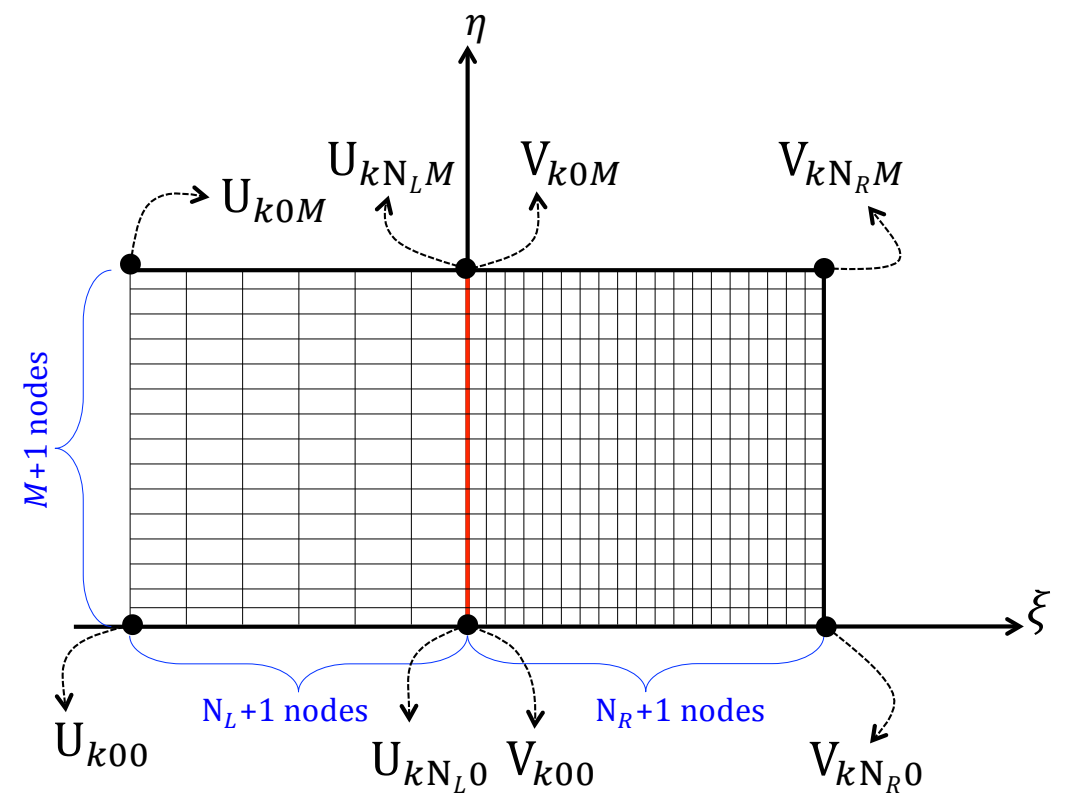

Figure 3: A schematic of the spatial mesh at $\tau=\tau_{k}$ and the indexing used in the arrangement of the numerical solutions.

The first derivative with respect to the $\xi$ direction is approximated by $D_{\xi} \mathbf{u}$, where $D_{\xi}$ is a so-called SBP operator decomposed as

$$
D_{\xi}=P_{\xi}^{-1} Q_{\xi}
$$


and $\mathbf{u}=\left[u_{0}, u_{1}, \cdots, u_{N}\right]^{T}$ is a smooth function evaluated at equidistant mesh points $\boldsymbol{\xi}=\left[\xi_{0}, \xi_{1}, \ldots, \xi_{N}\right]^{T}$. The matrix $P_{\xi}$ is symmetric positive definite, and $Q_{\xi}$ is almost skew-symmetric, satisfying

$$
Q_{\xi}+Q_{\xi}^{T}=E_{1}-E_{0}=B_{\xi}=\operatorname{diag}(-1,0, \ldots, 0,1) .
$$

In (18), $E_{0}=\operatorname{diag}(1,0, \ldots, 0)$ and $E_{1}=\operatorname{diag}(0, \ldots, 0,1)$, of size $N$. The directions $\eta$ and $\tau$ are discretized in the same way i.e. $D_{\tau}=P_{\tau}^{-1} Q_{\tau}$ and $D_{\eta}=P_{\eta}^{-1} Q_{\eta}$. We use the same SBP operators in the $\eta$ and $\tau$ directions for the left and right problems.

A first derivative SBP operator is a $2 s$-order accurate central difference operator which is modified close to the boundaries such that it becomes one-sided. Together with a diagonal norm $P$, the boundary closure is $s$-order accurate, making a stable first order approximation of a hyperbolic problem $s+1$ order accurate globally [4, 9]. For more details on non-standard SBP operators see [14, 15, 16, 17].

The extension of finite difference operators to multiple dimensions including the time discretization $[7,8]$, is done by arranging the one-dimensional SBP operators in a tensor product fashion as

$$
\begin{aligned}
& \left(\mathcal{D}_{\tau}\right)_{L, R}=D_{\tau} \otimes\left(I_{\xi}\right)_{L, R} \otimes I_{\eta} \otimes I \\
& \left(\mathcal{D}_{\xi}\right)_{L, R}=I_{\tau} \otimes\left(D_{\xi}\right)_{L, R} \otimes I_{\eta} \otimes I \\
& \left(\mathcal{D}_{\eta}\right)_{L, R}=I_{\tau} \otimes\left(I_{\xi}\right)_{L, R} \otimes D_{\eta} \otimes I
\end{aligned}
$$

In (19), $\otimes$ represents the Kronecker product [13] defined as

$$
A \otimes B=\left[\begin{array}{ccc}
a_{11} B & \ldots & a_{1 n} B \\
\vdots & \ddots & \vdots \\
a_{m 1} B & \ldots & a_{m n} B
\end{array}\right]
$$

for the $m \times n$ matrix $A=\left\{a_{i j}\right\}$ and $B$ of any size. In (19) and in the remainder of this article, all matrices in the first position are of size $(K+1) \times(K+1)$, the second position $\left(N_{L, R}+1\right) \times\left(N_{L, R}+1\right)$, the third position $(M+1) \times(M+1)$ and the fourth position $l \times l$. Additionally, $I_{\tau},\left(I_{\xi}\right)_{L, R}, I_{\eta}$ and $I$ denote identity matrices with a size consistent with their position in the Kronecker product.

Prior to the discretization of (9), we use the splitting technique in [18] required for problems with variable coefficients. The discrete version of (9) including a weak interface condition, a weak initial condition and weak far-field boundary 
conditions is

$$
\begin{aligned}
\frac{1}{2}\left[\left(\mathcal{D}_{\tau}\right)_{L} \mathbf{J}_{L} \mathbf{U}+\mathbf{J}_{L}\left(\mathcal{D}_{\tau}\right)_{L} \mathbf{U}+\left(\mathbf{J}_{L}\right)_{\tau} \mathbf{U}\right. & +\left(\mathcal{D}_{\xi}\right)_{L} \mathbf{A}_{L} \mathbf{U}+\mathbf{A}_{L}\left(\mathcal{D}_{\xi}\right)_{L} \mathbf{U}+\left(\mathbf{A}_{L}\right)_{\xi} \mathbf{U} \\
& \left.+\left(\mathcal{D}_{\eta}\right)_{L} \mathbf{B}_{L} \mathbf{U}+\mathbf{B}_{L}\left(\mathcal{D}_{\eta}\right)_{L} \mathbf{U}+\left(\mathbf{B}_{L}\right)_{\eta} \mathbf{U}\right] \\
& =\left(\mathcal{P}_{I}\right)_{L}^{-1} \Sigma_{L}(\mathbf{U}-\mathbf{V}) \\
& +\left(\mathcal{P}_{\tau}\right)_{L}^{-1}\left(\Sigma_{\tau}\right)_{L}\left(\mathbf{U}-\mathbf{f}_{L}\right) \\
& +\left(\mathcal{P}_{F B}\right)_{L}^{-1}\left(\Sigma_{F B}\right)_{L}\left(\mathbf{U}-\mathbf{U}_{\infty}\right), \\
\frac{1}{2}\left[\left(\mathcal{D}_{\tau}\right)_{R} \mathbf{J}_{R} \mathbf{V}+\mathbf{J}_{R}\left(\mathcal{D}_{\tau}\right)_{R} \mathbf{V}+\left(\mathbf{J}_{R}\right)_{\tau} \mathbf{V}\right. & +\left(\mathcal{D}_{\xi}\right)_{R} \mathbf{A}_{R} \mathbf{V}+\mathbf{A}_{R}\left(\mathcal{D}_{\xi}\right)_{R} \mathbf{V}+\left(\mathbf{A}_{R}\right)_{\xi} \mathbf{V} \\
& \left.+\left(\mathcal{D}_{\eta}\right)_{R} \mathbf{B}_{R} \mathbf{V}+\mathbf{B}_{R}\left(\mathcal{D}_{\eta}\right)_{R} \mathbf{V}+\left(\mathbf{B}_{R}\right)_{\eta} \mathbf{V}\right] \\
& =\left(\mathcal{P}_{I}\right)_{R}^{-1} \Sigma_{R}(\mathbf{V}-\mathbf{U}) \\
& +\left(\mathcal{P}_{\tau}\right)_{R}^{-1}\left(\Sigma_{\tau}\right)_{R}\left(\mathbf{V}-\mathbf{f}_{R}\right) \\
& +\left(\mathcal{P}_{F B}\right)_{R}^{-1}\left(\Sigma_{F B}\right)_{R}\left(\mathbf{V}-\mathbf{V}_{\infty}\right) .
\end{aligned}
$$

In (21), the terms including $\Sigma_{L, R},\left(\Sigma_{\tau}\right)_{L, R}$ and $\left(\Sigma_{F B}\right)_{L, R}$ are penalty terms related to the weak interface treatments, weak initial and far-field boundary conditions, respectively. We have already, in [1], given a thorough analysis for the weak initial and far-field boundary conditions in terms of conservation and stability. Hence, we only display these two terms in (21) for completeness without defining and resolving them further in this article. Instead, we focus on the weak interface conditions where $\left(\mathcal{P}_{I}\right)_{L, R}^{-1}=\left(I_{\tau} \otimes\left(P_{\xi}\right)_{L, R}^{-1} E_{0} \otimes I_{\eta} \otimes I\right)$ and choose $\Sigma_{L, R}$ based on conservation and stability requirements.

Moreover, in (21), $\mathbf{J}_{L, R}, \mathbf{A}_{L, R}, \mathbf{B}_{L, R},\left(\mathbf{A}_{L, R}\right)_{\xi}$ and $\left(\mathbf{B}_{L, R}\right)_{\eta}$ are block diagonal matrices approximating $J_{L, R}, A_{L, R}, B_{L, R},\left(A_{L, R}\right) \xi$ and $\left(B_{L, R}\right)_{\eta}$ pointwise. As an example, we use the following approximations for $\mathbf{J}_{L}, \mathbf{A}_{L}$ and $\mathbf{B}_{L}$,

$$
\begin{aligned}
\mathbf{J}_{L} & =\left(\overline{x_{\xi} y_{\eta}}\right)_{L}-\left(\overline{x_{\eta} y_{\xi}}\right)_{L} \\
\mathbf{A}_{L} & =\left(\overline{J \xi_{t}}\right)_{L}+\left(\overline{J \xi_{x}}\right)_{L}\left[I_{\tau} \otimes\left(I_{\xi}\right)_{L} \otimes I_{\eta} \otimes \hat{A}\right]+\left(\overline{J \xi_{y}}\right)_{L}\left[I_{\tau} \otimes\left(I_{\xi}\right)_{L} \otimes I_{\eta} \otimes \hat{B}\right], \\
\mathbf{B}_{L} & =\left(\overline{J \eta_{t}}\right)_{L}+\left(\overline{J \eta_{x}}\right)_{L}\left[I_{\tau} \otimes\left(I_{\xi}\right)_{L} \otimes I_{\eta} \otimes \hat{A}\right]+\left(\overline{J \eta_{y}}\right)_{L}\left[I_{\tau} \otimes\left(I_{\xi}\right)_{L} \otimes I_{\eta} \otimes \hat{B}\right],
\end{aligned}
$$

where the bar sign indicates a numerical approximation. We use

$$
\begin{array}{rlrl}
\left(\overline{x_{\xi} y_{\eta}}\right)_{L} & =\operatorname{diag}\left[\left(\mathcal{D}_{\eta}\right)_{L} Z^{(1)}\right], & \left(\overline{x_{\eta} y_{\xi}}\right)_{L} & =\operatorname{diag}\left[\left(\mathcal{D}_{\xi}\right)_{L} Z^{(2)}\right], \\
\left(\overline{J \xi_{t}}\right)_{L} & =\operatorname{diag}\left[\left(\mathcal{D}_{\tau}\right)_{L} Z^{(2)}-\left(\mathcal{D}_{\xi}\right)_{L} Z^{(3)}\right],\left(\overline{J \eta_{t}}\right)_{L}=\operatorname{diag}\left[\left(\mathcal{D}_{\xi}\right)_{L} Z^{(3)}-\left(\mathcal{D}_{\tau}\right)_{L} Z^{(1)}\right], \\
\left(\overline{J \xi_{x}}\right)_{L} & =\operatorname{diag}\left[\left(\mathcal{D}_{\eta}\right)_{L} \mathbf{y}\right], & \left(\overline{J \xi_{y}}\right)_{L} & =-\operatorname{diag}\left[\left(\mathcal{D}_{\eta}\right)_{L} \mathbf{x}\right], \\
\left(\overline{J \eta_{x}}\right)_{L} & =-\operatorname{diag}\left[\left(\mathcal{D}_{\xi}\right)_{L} \mathbf{y}\right], & \left(\overline{J \eta_{y}}\right)_{L} & =-\operatorname{diag}\left[\left(\mathcal{D}_{\xi}\right)_{L} \mathbf{x}\right],
\end{array}
$$


where $\mathbf{x}, \mathbf{y}$ are the $\mathbf{x}$ and $\mathrm{y}$ coordinates of the left mesh in the Cartesian coordinate system, arranged as a vector consistent to (16). In (23), we use

$$
Z^{(1)}=\operatorname{diag}(\mathbf{y})\left[\left(\mathcal{D}_{\xi}\right)_{L} \mathbf{x}\right], \quad Z^{(2)}=\operatorname{diag}(\mathbf{y})\left[\left(\mathcal{D}_{\eta}\right)_{L} \mathbf{x}\right], \quad Z^{(3)}=\operatorname{diag}(\mathbf{y})\left[\left(\mathcal{D}_{\tau}\right)_{L} \mathbf{x}\right] .
$$

Finally, the following relations are used

$$
\left(\mathbf{J}_{L, R}\right)_{\tau}=\left(\mathcal{D}_{\tau}\right)_{L, R} \mathbf{J}_{L, R}, \quad\left(\mathbf{A}_{L, R}\right)_{\xi}=\left(\mathcal{D}_{\xi}\right)_{L, R} \mathbf{A}_{L, R}, \quad\left(\mathbf{B}_{L, R}\right)_{\eta}=\left(\mathcal{D}_{\eta}\right)_{L, R} \mathbf{B}_{L, R} .
$$

The metric terms and approximations for the right sub-domain are handled in a similar way. For more details about the numerical approximation of the metric terms, see $[1,23]$.

Remark 2. By using the definitions given in (23) and (25), the Numerical Geometric Conservation Law (NGCL) holds and gives

$$
\left(\mathbf{J}_{L, R}\right)_{\tau}+\left(\mathbf{A}_{L, R}\right)_{\xi}+\left(\mathbf{B}_{L, R}\right)_{\eta}=\left(\mathcal{D}_{\tau} \mathbf{J}\right)_{L, R}+\left(\mathcal{D}_{\xi} \mathbf{A}\right)_{L, R}+\left(\mathcal{D}_{\eta} \mathbf{B}\right)_{L, R}=0
$$

As a consequence of using SBP in time, the temporal and spatial operators commute and the proof of (26) directly follows. See [1] for the proof.

Remark 3. Since the NGCL holds, the zeroth order terms on the left hand sides of (21) can be removed. In (29) and (33) below, we kept the zeroth order terms to be consistent with (21) and in order to compare with the continuous result in (12).

We end this subsection with a discussion on free-stream preservation. To this end, consider the left problem in (21), multiply with 2 and ignore the penalty terms. This gives

$$
\begin{aligned}
\left(\mathcal{D}_{\tau}\right)_{L} \mathbf{J}_{L} \mathbf{U}+\mathbf{J}_{L}\left(\mathcal{D}_{\tau}\right)_{L} \mathbf{U}+\left(\mathbf{J}_{L}\right)_{\tau} \mathbf{U} & +\left(\mathcal{D}_{\xi}\right)_{L} \mathbf{A}_{L} \mathbf{U}+\mathbf{A}_{L}\left(\mathcal{D}_{\xi}\right)_{L} \mathbf{U}+\left(\mathbf{A}_{L}\right)_{\xi} \mathbf{U} \\
& +\left(\mathcal{D}_{\eta}\right)_{L} \mathbf{B}_{L} \mathbf{U}+\mathbf{B}_{L}\left(\mathcal{D}_{\eta}\right)_{L} \mathbf{U}+\left(\mathbf{B}_{L}\right)_{\eta} \mathbf{U}=0 .
\end{aligned}
$$

By inserting a constant solution e.g. $\mathbf{U}=\mathbf{1}$, where $\mathbf{1}$ is a vector of the same size as $\mathbf{U}$ with all elements equal to one, we obtain

$$
\left(\mathcal{D}_{\tau}\right)_{L} \mathbf{J}_{L} \mathbf{1}+\left(\mathbf{J}_{L}\right)_{\tau} \mathbf{1}+\left(\mathcal{D}_{\xi}\right)_{L} \mathbf{A}_{L} \mathbf{1}+\left(\mathbf{A}_{L}\right)_{\xi} \mathbf{1}+\left(\mathcal{D}_{\eta}\right)_{L} \mathbf{B}_{L} \mathbf{1}+\left(\mathbf{B}_{L}\right)_{\eta} \mathbf{1}=0
$$

From (28), we clearly see that uniform flow is preserved thanks to the definitions in (22), (25) and (26) which guarantee that NGCL holds. 


\subsection{Discrete conservation}

To show that the scheme is conservative, we multiply (21) by $\phi_{L}$ and $\phi_{R}$ with compact support at the boundaries, and integrate numerically. Next, we use the SBP property (18) and the NGCL, and obtain

$$
\begin{gathered}
\frac{1}{2}\left[\mathbf { U } ^ { T } \mathcal { P } _ { L } \left(\left[\mathbf{J}_{L}\left(\mathcal{D}_{\tau}\right)_{L}+\left(\mathcal{D}_{\tau}\right)_{L} \mathbf{J}_{L}+\left(\mathbf{J}_{L}\right)_{\tau}\right] \phi_{L}+\left[\left(\mathbf{A}_{L}\left(D_{\xi}\right)_{L}+\left(D_{\xi}\right)_{L} \mathbf{A}_{L}+\left(\mathbf{A}_{L}\right) \xi\right] \phi_{L}+\right.\right.\right. \\
\left.\left.\left[\mathbf{B}_{L}\left(\mathcal{D}_{\eta}\right)_{L}+\left(\mathcal{D}_{\eta}\right)_{L} \mathbf{B}_{L}+\left(\mathbf{B}_{L}\right)_{\eta}\right] \phi_{L}\right)\right]^{T}+ \\
\frac{1}{2}\left[\mathbf { V } ^ { T } \mathcal { P } _ { R } \left(\left[\mathbf{J}_{R}\left(\mathcal{D}_{\tau}\right)_{R}+\left(\mathcal{D}_{\tau}\right)_{R} \mathbf{J}_{R}+\left(\mathbf{J}_{R}\right)_{\tau}\right] \phi_{R}+\left[\left(\mathbf{A}_{R}\left(\mathcal{D}_{\xi}\right)_{R}+\left(\mathcal{D}_{\xi}\right)_{R} \mathbf{A}_{R}+\left(\mathbf{A}_{R}\right)_{\xi}\right] \phi_{R}+\right.\right.\right. \\
\left.\left.\left[\mathbf{B}_{R}\left(\mathcal{D}_{\eta}\right)_{R}+\left(\mathcal{D}_{\eta}\right)_{R} \mathbf{B}_{R}+\left(\mathbf{B}_{R}\right)_{\eta}\right] \phi_{R}\right)\right]^{T}= \\
\phi_{L}^{T}\left(P_{\tau} \otimes E_{1} \otimes P_{\eta} \otimes I\right) \mathbf{A}_{L} \mathbf{U}-\phi_{L}^{T}\left(P_{\tau} \otimes E_{1} \otimes P_{\eta} \otimes I\right) \Sigma_{L}(\mathbf{U}-\mathbf{V}) \\
-\phi_{R}^{T}\left(P_{\tau} \otimes E_{0} \otimes P_{\eta} \otimes I\right) \mathbf{A}_{R} \mathbf{V}-\phi_{R}^{T}\left(P_{\tau} \otimes E_{0} \otimes P_{\eta} \otimes I\right) \Sigma_{R}(\mathbf{V}-\mathbf{U}),
\end{gathered}
$$

where $\mathcal{P}_{L, R}=P_{\tau} \otimes\left(P_{\xi}\right)_{L, R} \otimes P_{\eta} \otimes I$. The details of the derivations are given in Appendix B.

Considering $\phi_{L}=\phi_{R}:=\phi_{I}$ at the interface, takes the right hand side of (29) to

$$
\phi_{I}^{T}\left(P_{\tau} \otimes P_{\eta} \otimes I\right)\left[\mathbf{A}_{L_{I}} \mathbf{U}_{I}-\mathbf{A}_{R_{I}} \mathbf{V}_{I}+\left(\Sigma_{R_{I}}-\Sigma_{L_{I}}\right)\left(\mathbf{U}_{I}-\mathbf{V}_{I}\right)\right]
$$

where the subscript $I$ indicates interface. The matrices and vectors in (30), are now of size $(K+1)(M+1) l \times(K+1)(M+1) l$ and $(K+1)(M+1) l$, respectively.

As mentioned before, the matrices $\mathbf{A}_{L}$ and $\mathbf{A}_{R}$ include pointwise approximations to $A_{L}$ and $A_{R}$, respectively, as described in (22). This means that, we have $\mathbf{A}_{L_{I}}=\mathbf{A}_{R_{I}}:=\mathbf{A}_{\mathbf{I}}$, which corresponds to the continuous requirement in (11).

We use the decomposition $\mathbf{A}_{I}=\mathbf{X}_{I} \Lambda_{I} \mathbf{X}_{I}^{T}$ and choose $\Sigma_{L_{I}, R_{I}}=\mathbf{X}_{I} \tilde{\Sigma}_{L_{I}, R_{I}} \mathbf{X}_{I}^{T}$ where $\tilde{\Sigma}_{L_{I}, R_{I}}$ are diagonal and rewrite (30) as

$$
\phi_{I}^{T}\left(P_{\tau} \otimes P_{\eta} \otimes I\right)\left[\mathbf{X}_{I}\left(\Lambda_{I}-\tilde{\Sigma}_{L_{I}}+\tilde{\Sigma}_{R_{I}}\right) \mathbf{X}_{I}^{T}\right]\left(\mathbf{U}_{I}-\mathbf{V}_{I}\right) .
$$

In order to obtain a conservative scheme, $\tilde{\Sigma}_{L_{I}, R_{I}}$ must be chosen such that

$$
\Lambda_{I}-\tilde{\Sigma}_{L_{I}}+\tilde{\Sigma}_{R_{I}}=0
$$


holds, by which the right hand side of (29) vanishes and we get $\mathrm{F}$

$$
\begin{gathered}
\frac{1}{2}\left[\mathbf { U } ^ { T } \mathcal { P } _ { L } \left(\left[\mathbf{J}_{L}\left(\mathcal{D}_{\tau}\right)_{L}+\left(\mathcal{D}_{\tau}\right)_{L} \mathbf{J}_{L}+\left(\mathbf{J}_{L}\right)_{\tau}\right] \phi_{L}+\left[\left(\mathbf{A}_{L}\left(D_{\xi}\right)_{L}+\left(D_{\xi}\right)_{L} \mathbf{A}_{L}+\left(\mathbf{A}_{L}\right)_{\xi}\right] \phi_{L}+\right.\right.\right. \\
\left.\left.\left[\mathbf{B}_{L}\left(\mathcal{D}_{\eta}\right)_{L}+\left(\mathcal{D}_{\eta}\right)_{L} \mathbf{B}_{L}+\left(\mathbf{B}_{L}\right)_{\eta}\right] \phi_{L}\right)\right]^{T}+ \\
\frac{1}{2}\left[\mathbf { V } ^ { T } \mathcal { P } _ { R } \left(\left[\mathbf{J}_{R}\left(\mathcal{D}_{\tau}\right)_{R}+\left(\mathcal{D}_{\tau}\right)_{R} \mathbf{J}_{R}+\left(\mathbf{J}_{R}\right)_{\tau}\right] \phi_{R}+\left[\left(\mathbf{A}_{R}\left(\mathcal{D}_{\xi}\right)_{R}+\left(\mathcal{D}_{\xi}\right)_{R} \mathbf{A}_{R}+\left(\mathbf{A}_{R}\right)_{\xi}\right] \phi_{R}+\right.\right.\right. \\
\left.\left.\left[\mathbf{B}_{R}\left(\mathcal{D}_{\eta}\right)_{R}+\left(\mathcal{D}_{\eta}\right)_{R} \mathbf{B}_{R}+\left(\mathbf{B}_{R}\right)_{\eta}\right] \phi_{R}\right)\right]^{T}=0 .
\end{gathered}
$$

Equation (33) mimics the continuous weak conservative formulation in (12).

We summarize the result of this section in the following proposition.

Proposition 3. The interface procedure in (21) is conservative by the use of the metric terms in (22) and the interface penalty parameters satisfying (32).

\subsection{Stability}

To prove stability we apply the discrete energy method to (21), by multiplying the left and right problems with $\mathbf{U}^{T} \mathcal{P}_{L}$ and $\mathbf{V}^{T} \mathcal{P}_{R}$, respectively. We add the results to their transpose, use the SBP property (18), apply the NGCL given in Remark 2 and arrive at

$$
\begin{aligned}
& \mathbf{U}^{T}\left[B_{\tau} \otimes\left(P_{\xi}\right)_{L} \otimes P_{\eta} \otimes I\right] \mathbf{J}_{L} \mathbf{U}+\mathbf{U}^{T}\left[P_{\tau} \otimes\left(B_{\xi}\right)_{L} \otimes P_{\eta} \otimes I\right] \mathbf{A}_{L} \mathbf{U}+ \\
& \mathbf{U}^{T}\left[P_{\tau} \otimes\left(P_{\xi}\right)_{L} \otimes B_{\eta} \otimes I\right] \mathbf{B}_{L} \mathbf{U}=\mathbf{U}^{T}\left(P_{\tau} \otimes E_{1} \otimes P_{\eta} \otimes I\right) \Sigma_{L}(\mathbf{U}-\mathbf{V})+ \\
& (\mathbf{U}-\mathbf{V})^{T} \Sigma_{L}^{T}\left(P_{\tau} \otimes E_{1} \otimes P_{\eta} \otimes I\right) \mathbf{U} \\
& \mathbf{V}^{T}\left[B_{\tau} \otimes\left(P_{\xi}\right)_{R} \otimes P_{\eta} \otimes I\right] \mathbf{J}_{R} \mathbf{V}+\mathbf{V}^{T}\left[P_{\tau} \otimes\left(B_{\xi}\right)_{R} \otimes P_{\eta} \otimes I\right] \mathbf{A}_{R} \mathbf{V}+ \\
& \mathbf{V}^{T}\left[P_{\tau} \otimes\left(P_{\xi}\right)_{R} \otimes B_{\eta} \otimes I\right] \mathbf{B}_{R} \mathbf{V}=\mathbf{V}^{T}\left(P_{\tau} \otimes E_{0} \otimes P_{\eta} \otimes I\right) \Sigma_{R}(\mathbf{V}-\mathbf{U})+ \\
& (\mathbf{V}-\mathbf{U})^{T} \Sigma_{R}^{T}\left(P_{\tau} \otimes E_{0} \otimes P_{\eta} \otimes I\right) \mathbf{V} .
\end{aligned}
$$

Simplifying (34) and only keeping the terms at the interface give

$$
\begin{aligned}
& \|\mathbf{U}\|_{\left[E_{1} \otimes\left(P_{\xi}\right)_{L} \otimes P_{\eta} \otimes I\right] \mathbf{J}_{L}}^{2}-\|\mathbf{U}\|_{\left[E_{0} \otimes\left(P_{\xi}\right)_{L} \otimes P_{\eta} \otimes I\right] \mathbf{J}_{L}}^{2}=\mathbf{U}^{T}\left[P_{\tau} \otimes-E_{1} \otimes P_{\eta} \otimes I\right] \mathbf{A}_{L} \mathbf{U}+ \\
& \mathbf{U}^{T}\left(P_{\tau} \otimes E_{1} \otimes P_{\eta} \otimes I\right) \Sigma_{L}(\mathbf{U}-\mathbf{V})+(\mathbf{U}-\mathbf{V})^{T} \Sigma_{L}^{T}\left(P_{\tau} \otimes E_{1} \otimes P_{\eta} \otimes I\right) \mathbf{U}, \\
& \|\mathbf{V}\|_{\left[E_{1} \otimes\left(P_{\xi}\right)_{R} \otimes P_{\eta} \otimes I\right] \mathbf{J}_{R}}^{2}-\|\mathbf{V}\|_{\left[E_{0} \otimes\left(P_{\xi}\right)_{R} \otimes P_{\eta} \otimes I\right] \mathbf{J}_{R}}^{2}=\mathbf{V}^{T}\left[P_{\tau} \otimes E_{0} \otimes P_{\eta} \otimes I\right] \mathbf{A}_{R} \mathbf{V}+ \\
& \mathbf{V}^{T}\left(P_{\tau} \otimes E_{0} \otimes P_{\eta} \otimes I\right) \Sigma_{R}(\mathbf{V}-\mathbf{U})+(\mathbf{V}-\mathbf{U})^{T} \Sigma_{R}^{T}\left(P_{\tau} \otimes E_{0} \otimes P_{\eta} \otimes I\right) \mathbf{V} .
\end{aligned}
$$

The norms in (35) are defined by $\|\mathbf{W}\|_{H}^{2}=\mathbf{W}^{T} H \mathbf{W}$, where $\mathbf{W} \in\{\mathbf{U}, \mathbf{V}\}, H \in$ $\left\{\left[E_{1} \otimes\left(P_{\xi}\right)_{L, R} \otimes P_{\eta} \otimes I\right] J_{L, R},\left[E_{0} \otimes\left(P_{\xi}\right)_{L, R} \otimes P_{\eta} \otimes I\right] J_{L, R}\right\}$ and $H>0$. We add the 
two relations in (35) and only consider the terms at the interface. The decompositions $\mathbf{A}_{I}=\mathbf{X}_{I} \Lambda_{I} \mathbf{X}_{I}^{T}$ and $\Sigma_{L_{I}, R_{I}}=\mathbf{X}_{I} \tilde{\Sigma}_{L_{I}, R_{I}} \mathbf{X}_{I}^{T}$ lead to

$$
\begin{aligned}
& \|\mathbf{U}\|_{\left[E_{1} \otimes\left(P_{\xi}\right)_{L} \otimes P_{\eta} \otimes I\right] \mathbf{J}_{L}}^{+}\|\mathbf{V}\|_{\left[E_{1} \otimes\left(P_{\xi}\right)_{R} \otimes P_{\eta} \otimes I\right] \mathbf{J}_{R}}^{2}\|\mathbf{U}\|_{\left[E_{0} \otimes\left(P_{\xi}\right)_{L} \otimes P_{\eta} \otimes I\right]}^{2}+\|\mathbf{J}\|_{\left[E_{0} \otimes\left(P_{\xi}\right)_{R} \otimes P_{\eta} \otimes I\right] \mathbf{J}_{R}}^{2} \\
& +\left[\begin{array}{c}
\mathbf{X}_{I}^{T} \mathbf{U}_{I} \\
\mathbf{X}_{I}^{T} \mathbf{V}_{I}
\end{array}\right]^{T}\left[\begin{array}{cc}
P_{\tau} \otimes P_{\eta} \otimes I & 0 \\
0 & P_{\tau} \otimes P_{\eta} \otimes I
\end{array}\right]\left[\begin{array}{cc}
-\boldsymbol{\Lambda}_{I}+2 \tilde{\Sigma}_{L_{I}} & -\left(\tilde{\Sigma}_{L_{I}}+\tilde{\Sigma}_{R_{I}}\right) \\
-\left(\tilde{\Sigma}_{L_{I}}+\tilde{\Sigma}_{R_{I}}\right) & \boldsymbol{\Lambda}_{I}+2 \tilde{\Sigma}_{R_{I}}
\end{array}\right]\left[\begin{array}{l}
\mathbf{X}_{I}^{T} \mathbf{U}_{I} \\
\mathbf{X}_{I}^{T} \mathbf{V}_{I}
\end{array}\right] .
\end{aligned}
$$

By considering the conservation requirement (32), (36) becomes

$$
\begin{aligned}
& \|\mathbf{U}\|_{\left[E_{1} \otimes\left(P_{\xi}\right)_{L} \otimes P_{\eta} \otimes I\right]}^{2}+\left.\mathbf{J}_{L}|| \mathbf{V}\left\|_{\left[E_{1} \otimes\left(P_{\xi}\right)_{R} \otimes P_{\eta} \otimes I\right] \mathbf{J}_{R}}^{2}=\right\| \mathbf{U}\right|_{\left[E_{0} \otimes\left(P_{\xi}\right)_{L} \otimes P_{\eta} \otimes I\right]} ^{2}+\|\mathbf{J}\|_{\left[E_{0} \otimes\left(P_{\xi}\right)_{R} \otimes P_{\eta} \otimes I\right] \mathbf{J}_{R}}^{2} \\
& +\left[\mathbf{X}_{I}^{T}\left(\mathbf{U}_{I}-\mathbf{V}_{I}\right)\right]^{T}\left(P_{\tau} \otimes P_{\eta} \otimes I\right)\left[-\boldsymbol{\Lambda}_{I}+2 \tilde{\Sigma}_{L_{I}}\right] \mathbf{X}_{I}^{T}\left(\mathbf{U}_{I}-\mathbf{V}_{I}\right) .
\end{aligned}
$$

From (37), we conclude that the following conditions lead to a stable and conservative interface treatment

$$
\tilde{\Sigma}_{L_{I}} \leq \Lambda_{I} / 2
$$

We summarize the result of this section in the following proposition.

Proposition 4. The scheme in (21) subject to (32) and (38) lead to a stable and conservative interface procedure.

We choose $\tilde{\Sigma}_{L_{I}}=\left(\Lambda_{I}-\left|\Lambda_{I}\right|\right) / 2$ and $\tilde{\Sigma}_{R_{I}}=-\left(\Lambda_{I}+\left|\Lambda_{I}\right|\right) / 2$ by which (32) and (38) are satisfied and (37) becomes

$$
\begin{array}{r}
\left\|\left.\mathbf{U}\right|_{\left[E_{1} \otimes\left(P_{\xi}\right)_{L} \otimes P_{\eta} \otimes I\right] \mathbf{J}_{L}} ^{2}|| \mathbf{V}\right\|_{\left[E_{1} \otimes\left(P_{\xi}\right)_{R} \otimes P_{\eta} \otimes I\right] \mathbf{J}_{R}}^{2}=\|\mathbf{U}\|_{\left[E_{0} \otimes\left(P_{\xi}\right)_{L} \otimes P_{\eta} \otimes I\right] \mathbf{J}_{L}}^{2}+\| \mathbf{V}||_{\left[E_{0} \otimes\left(P_{\xi}\right)_{R} \otimes P_{\eta} \otimes I\right] \mathbf{J}_{R}}^{2} \\
-\left(\mathbf{U}_{I}-\mathbf{V}_{I}\right)^{T}\left(P_{\tau} \otimes P_{\eta} \otimes I\right) \underbrace{\mathbf{X}_{I}\left|\boldsymbol{\Lambda}_{I}\right| \mathbf{X}_{I}^{T}}_{=\left|\mathbf{A}_{I}\right|}\left(\mathbf{U}_{I}-\mathbf{V}_{I}\right) .
\end{array}
$$

Remark 4. With the recently derived interface treatment, it can be shown that the scheme is strongly stable and mimics the continuous estimate in (15). This was previously done in [1] and we do not repeat those derivations here.

\section{Numerical experiments}

We will exemplify the theoretical developments above by choosing applications in aeroacoustics using the linearized Euler equations and electro-magnetics using the Maxwell equations. 


\subsection{The linearized Euler equations}

We consider the two-dimensional constant coefficient symmetrized Euler equations [19] given by

$$
W_{t}+\hat{A} W_{x}+\hat{B} W_{y}=0,(x, y) \in \Omega_{L, R}(t), t \in[0, T],
$$

where

$$
W=\left[\frac{\bar{c} \rho}{\sqrt{\gamma} \bar{\rho}}, u, v, \frac{\theta}{\bar{c} \sqrt{\gamma(\gamma-1)}}\right]^{T} .
$$

In (41), $W \in\{U, V\}$, and $\rho, u, v$ and $\theta$ are respectively the perturbations in density, the $x$ and $y$ velocity components and the temperature. An equation of state of the form $\gamma p=\bar{\rho} \theta+\rho \bar{\theta}$, where $p$ is the perturbation in pressure and $\gamma$ is the ratio of specific heats, completes the system (40). Moreover, the bar sign denotes the reference state around which we have linearized. The matrices in (40) are

$$
\hat{A}=\left[\begin{array}{cccc}
\bar{u} & \bar{c} / \sqrt{\gamma} & 0 & 0 \\
\bar{c} / \sqrt{\gamma} & \bar{u} & 0 & \sqrt{\frac{\gamma-1}{\gamma}} \bar{c} \\
0 & 0 & \bar{u} & 0 \\
0 & \sqrt{\frac{\gamma-1}{\gamma}} \bar{c} & 0 & \bar{u}
\end{array}\right], \hat{B}=\left[\begin{array}{cccc}
\bar{v} & 0 & \bar{c} / \sqrt{\gamma} & 0 \\
0 & \bar{v} & 0 & 0 \\
\bar{c} / \sqrt{\gamma} & 0 & \bar{v} & \sqrt{\frac{\gamma-1}{\gamma}} \bar{c} \\
0 & 0 & \sqrt{\frac{\gamma-1}{\gamma}} \bar{c} & \bar{v}
\end{array}\right] .
$$

We prescribe $\gamma=1.4, \bar{c}=2, \bar{\rho}=1$ and consider a subsonic background velocity field $(\bar{u}, \bar{v})=(1,1)$.

The geometries $\Omega_{L, R}(t)$ are described by

$$
\Omega_{L}(t):\left\{\begin{array}{l}
x_{w_{L}}(y)=-1-0.1 \sin (2 \pi y), \\
x_{I}(t, y)=-0.1[\sin (\pi t)+\cos (2 \pi t) \sin (2 \pi y)], \\
y_{s_{L}}(t, x)=-0.05 \sin \left[2 \pi\left(x_{I}(t)-x\right) /\left(x_{I}(t)+1\right)\right], \\
y_{n_{L}}(t, x)=1-0.05 \sin \left[2 \pi\left(x_{I}(t)-x\right) /\left(x_{I}(t)+1\right)\right],
\end{array}\right.
$$

and 


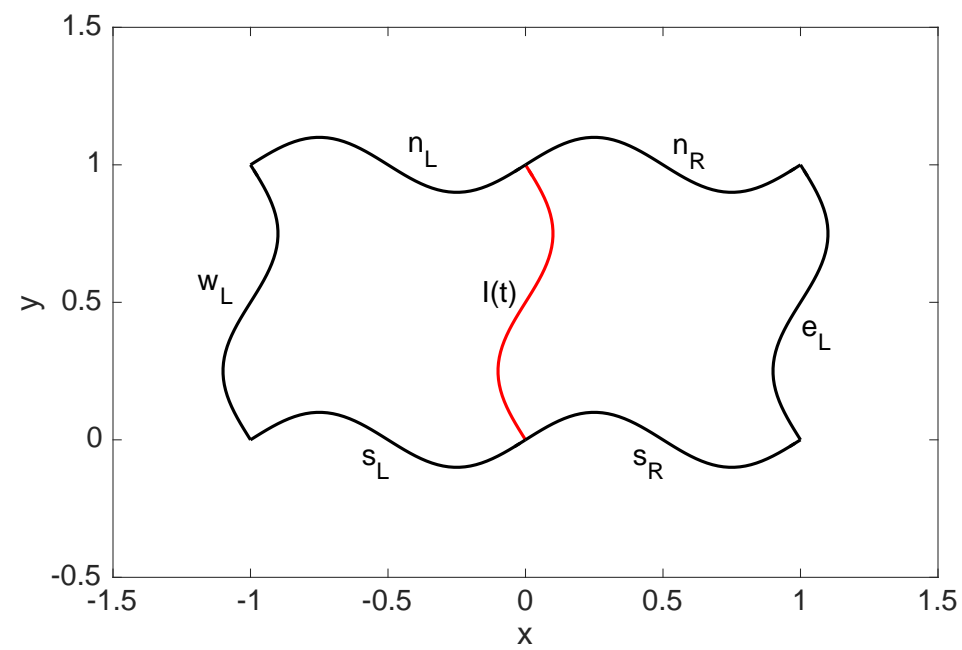

Figure 4: A schematic of $\Omega_{L, R}$ and the boundaries

$$
\Omega_{R}(t):\left\{\begin{array}{l}
x_{e_{R}}(y)=1-0.1 \sin (2 \pi y), \\
x_{I}(t, y)=-0.1[\sin (\pi t)+\cos (2 \pi t) \sin (2 \pi y)], \\
y_{s_{R}}(t, x)=-0.05 \sin \left[2 \pi\left(x-x_{I}(t)\right) /\left(1-x_{I}(t)\right)\right], \\
x_{n_{R}}(t, y)=1+0.05 \sin \left[2 \pi\left(x-x_{I}(t)\right) /\left(1-x_{I}(t)\right)\right] .
\end{array}\right.
$$

The notations $w_{L}, e_{R}, s_{L, R}, n_{L, R}$ and $I$ are illustrated in Figure 4.

\subsubsection{Accuracy}

To determine the accuracy of our numerical approximations, we use the method of manufactured solution with the reference solution $W_{\infty}$

$$
W_{\infty}=[\sin (\alpha x-\beta t), \cos (\alpha x-\beta t), \sin (\beta y-\alpha t), \cos (\beta y-\alpha t)]^{T}
$$

where $W_{\infty} \in\left\{U_{\infty}, V_{\infty}\right\}$ is used to form a forcing function, boundary and initial data to (40). In (45), $\alpha, \beta$ are arbitrary constants.

We examine the scheme for SBP operators of order $2 s$ in the interior and $s$ close to the spatial boundaries, where $s \in\{1,2,3\}$. The fifth order accurate SBP operator i.e. SBP84, with a sufficiently large $K$, is used in time. For computational reasons, a multi-block formulation in time is used. In the multi-block formulation, the time interval is divided into an arbitrary number of smaller blocks where the 
final solution of each block is weakly imposed as initial data to the next block. By using this technique, we make the computations faster and more efficient. The SBP multi-block technique is outline in [8].

The rates of convergence $q$ are given by

$$
q=\frac{\log \frac{\left\|W_{\infty}^{(1)}-W^{(1)}\right\|_{\mathcal{P}_{L, R}}}{\left\|W_{\infty}^{(2)}-W^{(2)}\right\|_{\mathcal{P}_{L, R}}}}{\log \frac{\left(N_{L, R}^{(1)}+1\right)\left(M^{(1)}+1\right)}{\left(N_{L, R}^{(2)}+1\right)\left(M^{(2)}+1\right)}} .
$$

where superscripts (1) and (2) denote two mesh levels with $\left(N_{L, R}^{(1)}+1\right) \times\left(M^{(1)}+1\right)$ and $\left(N_{L, R}^{(2)}+1\right) \times\left(M^{(2)}+1\right)$ grid points, respectively. In (46), $\mathcal{P}_{L, R}=P_{\tau} \otimes\left(P_{\xi}\right)_{L, R} \otimes$ $P_{\eta} \otimes I$ and $W \in\{U, V\}$. In Tables 1-3, the convergence rates are calculated and shown for a series of mesh refinements in space.

\begin{tabular}{c|c|c|c|c|c}
$N_{L, R}, M$ & 21 & 31 & 41 & 51 & 61 \\
\hline$\rho$ & 2.3963 & 2.0848 & 2.0380 & 2.0240 & 2.0161 \\
\hline$u$ & 2.1238 & 2.0399 & 2.0260 & 2.0193 & 2.0150 \\
\hline$v$ & 2.0781 & 2.0225 & 2.0182 & 2.0109 & 2.0072 \\
\hline$p$ & 2.4717 & 2.1218 & 2.0509 & 2.0295 & 2.0192
\end{tabular}

Table 1: Convergence rates at $\mathrm{T}=1$, for a sequence of mesh refinements, SBP21 in space, SBP84 in time ( $\mathrm{K}=801), \alpha=5, \beta=10$

\begin{tabular}{c|c|c|c|c|c}
$N_{L, R}, M$ & 21 & 31 & 41 & 51 & 61 \\
\hline$\rho$ & 3.0899 & 3.0997 & 3.0563 & 3.0494 & 3.0443 \\
\hline$u$ & 3.3222 & 3.0938 & 3.0213 & 3.0219 & 3.0070 \\
\hline$v$ & 2.2905 & 3.0968 & 3.1173 & 3.1157 & 3.1101 \\
\hline$p$ & 3.0569 & 3.1541 & 3.2048 & 3.1710 & 3.1138
\end{tabular}

Table 2: Convergence rates at $\mathrm{T}=1$, for a sequence of mesh refinements, SBP42 in space, SBP84 in time $(\mathrm{K}=801), \alpha=5, \beta=10$ 


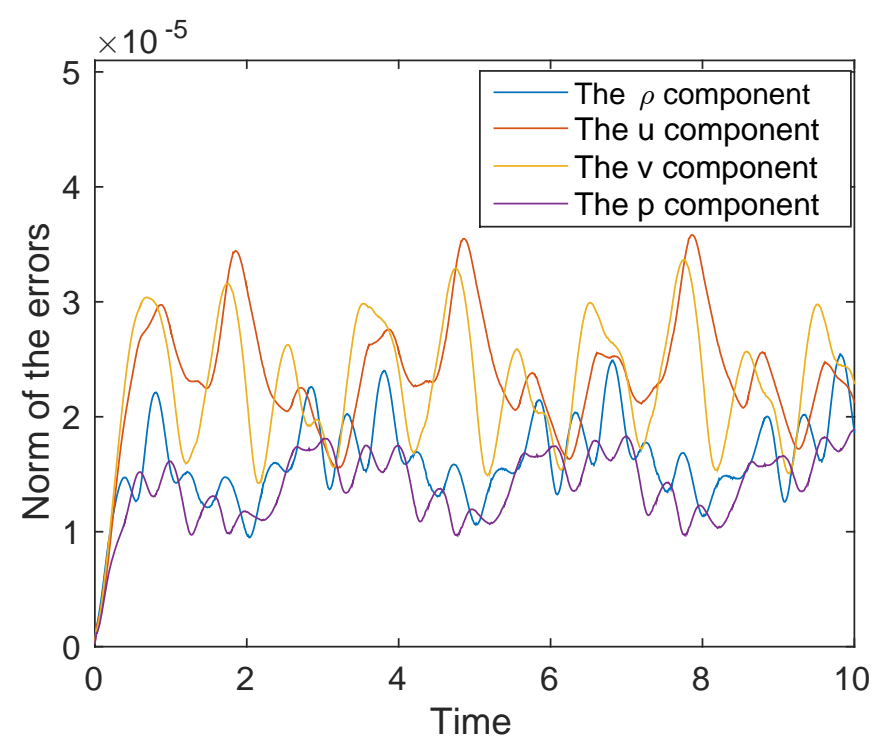

Figure 5: Long-time calculations for the Euler equations, error norm versus time, $\mathrm{T}=10$

\begin{tabular}{c|c|c|c|c|c}
$N_{L, R}, M$ & 21 & 31 & 41 & 51 & 61 \\
\hline$\rho$ & 3.8734 & 4.2147 & 4.3433 & 4.4085 & 4.4594 \\
\hline$u$ & 3.8653 & 3.8543 & 4.1876 & 4.3426 & 4.4153 \\
\hline$v$ & 4.1015 & 4.1432 & 4.1320 & 4.1643 & 4.2150 \\
\hline$p$ & 3.4794 & 3.5807 & 4.0005 & 4.1717 & 4.2293
\end{tabular}

Table 3: Convergence rates at $\mathrm{T}=1$, for a sequence of mesh refinements, SBP63 in space, SBP84 in time ( $\mathrm{K}=801), \alpha=5, \beta=10$

As seen in Tables 1-3, the design convergence rates are obtained, according to the theory in $[4,9,20,21]$.

\subsubsection{Long-time calculations}

Long time calculations until $T=10$ are presented in Figure 5. We have used a third order SBP operator in space with $\left(N_{L, R}=M=41\right)$ and a fifth order SBP operator in time with $K=1001$. The manufactured solution in (45) with $\alpha=1$ and $\beta=1$ is chosen to quantify the error. The results in Figure 5, show that the error is bounded in time which should be the case according to the theory in [31]. 

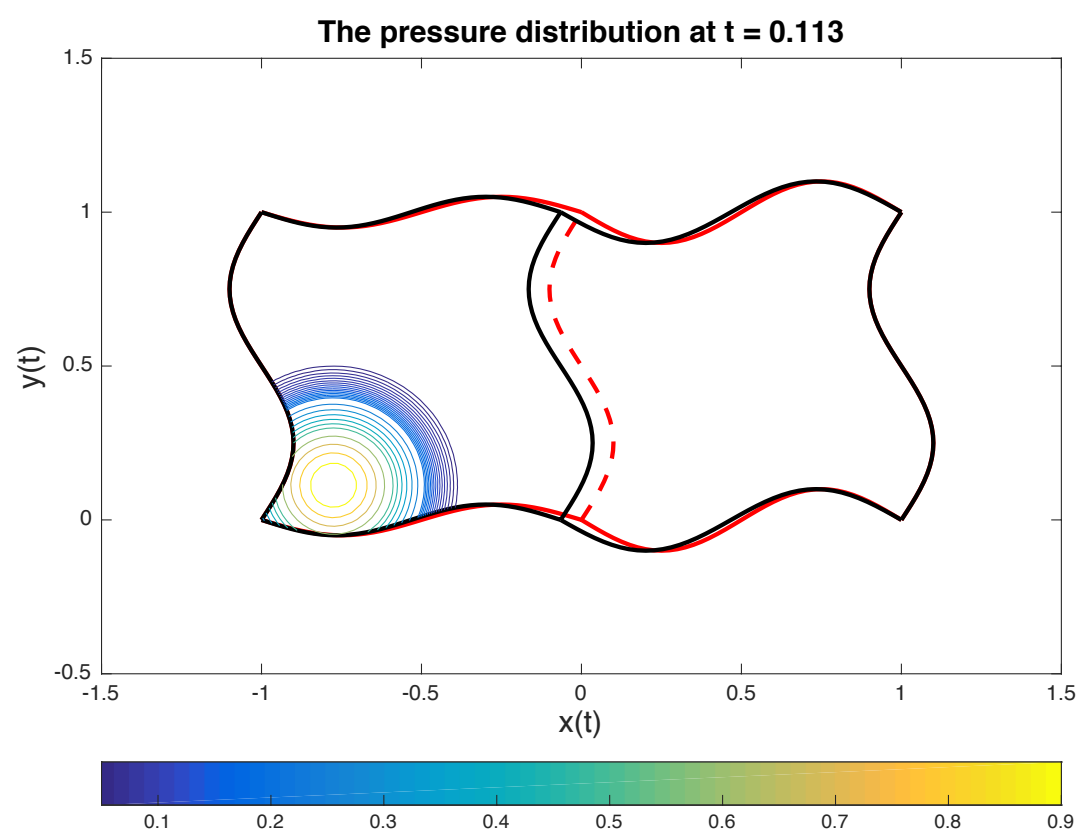

Figure 6: The pressure distribution.

\subsubsection{An application}

We again consider (40) but now with data taken from a manufactured solution of the form

$$
W_{\infty}=\left[0,0,0, e^{-20\left((x+1-2 t)^{2}+(y-t)^{2}\right)}\right]^{T} .
$$

Characteristic boundary conditions are used. Moreover, we construct a grid consisting of $41 \times 41$ spatial nodes in each sub-domain and 81 points in time for $t \in[0,1]$. Third and fifth order accurate SBP operators in space and time, respectively, are used.

The pressure distribution at different times (the red and/or dashed lines corresponding to $\Omega_{L, R}(0)$ ) are shown in Figures 6-13. As shown in the figures, the pressure pulse passes across the interface smoothly and moves out of the domain as time progresses.

\subsection{The Maxwell equations}

The Maxwell equations in two spatial dimensions are given by

$$
\begin{aligned}
\mu H_{t} & =-\nabla \times E, & \varepsilon E_{t} & =\nabla \times H-J, \\
\nabla \cdot \varepsilon E & =\rho, & \nabla \cdot \mu H & =0
\end{aligned}
$$



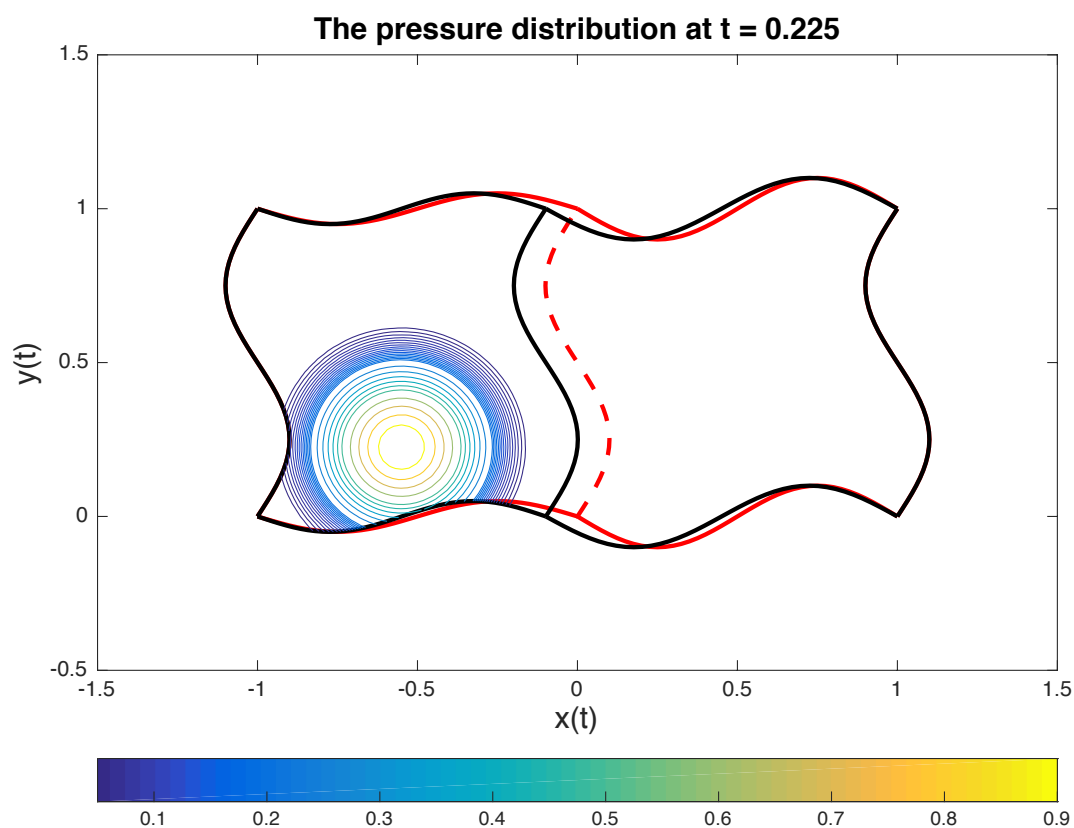

Figure 7: The pressure distribution.
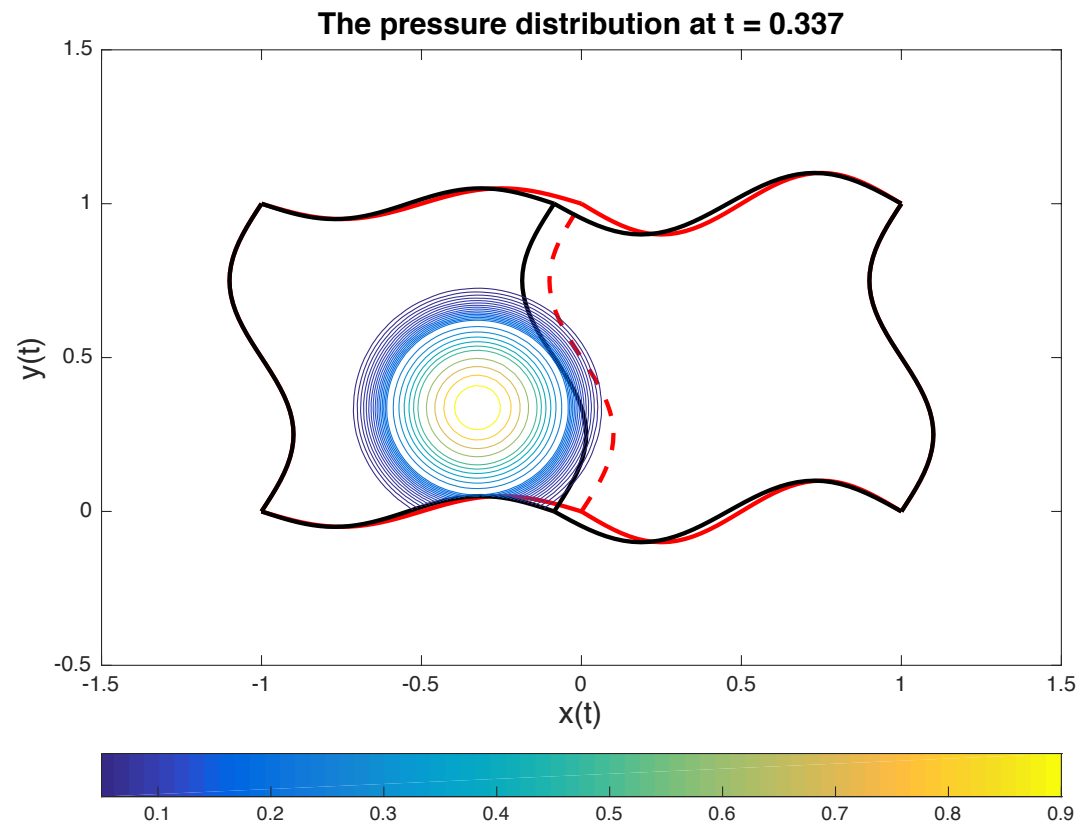

Figure 8: The pressure distribution. 

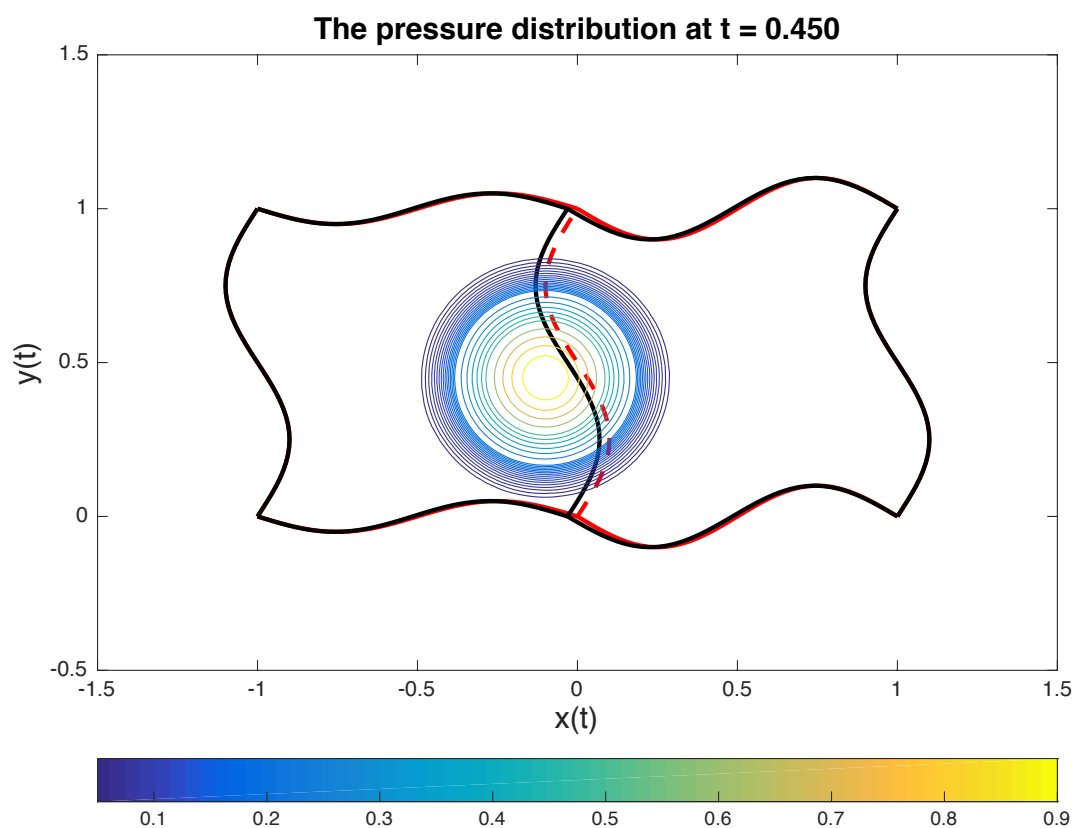

Figure 9: The pressure distribution.

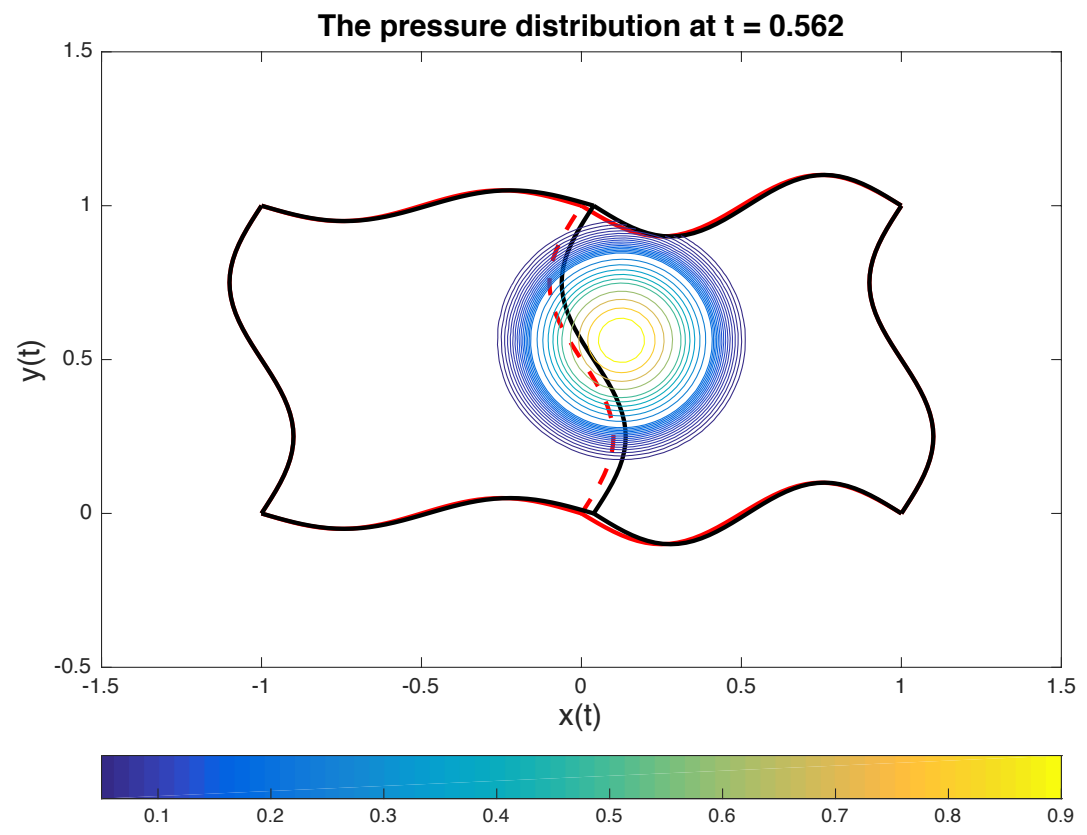

Figure 10: The pressure distribution. 

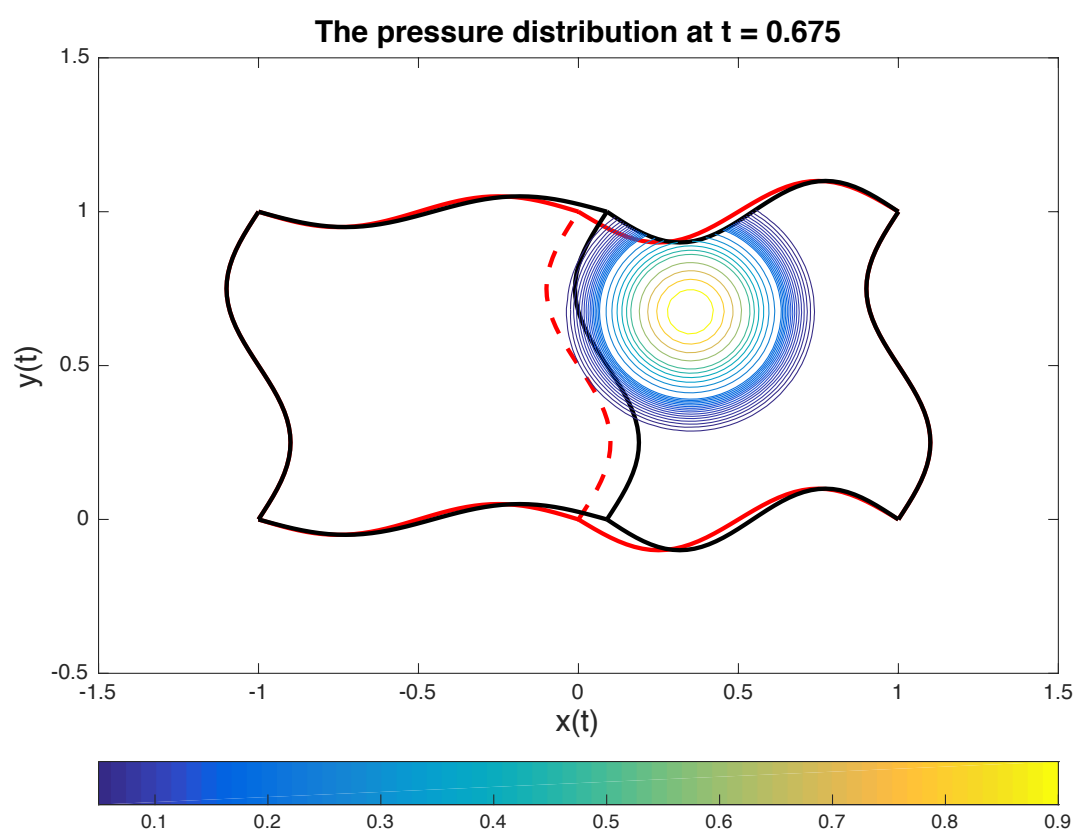

Figure 11: The pressure distribution.

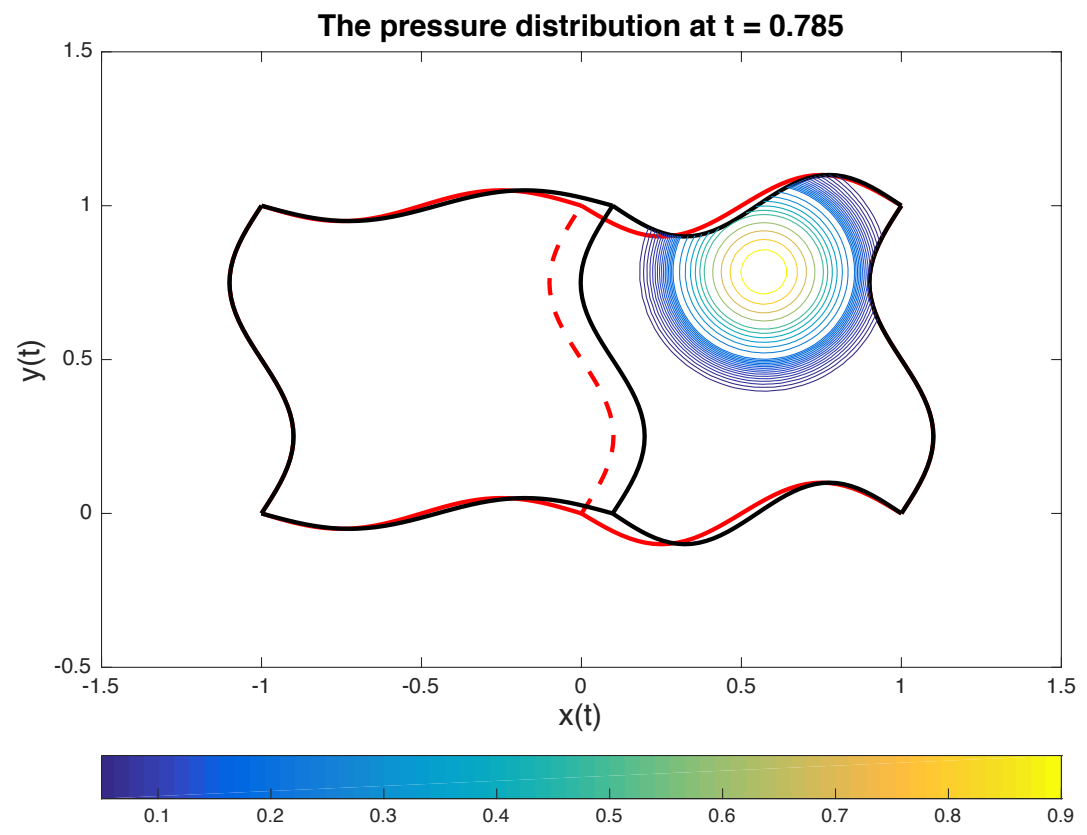

Figure 12: The pressure distribution. 

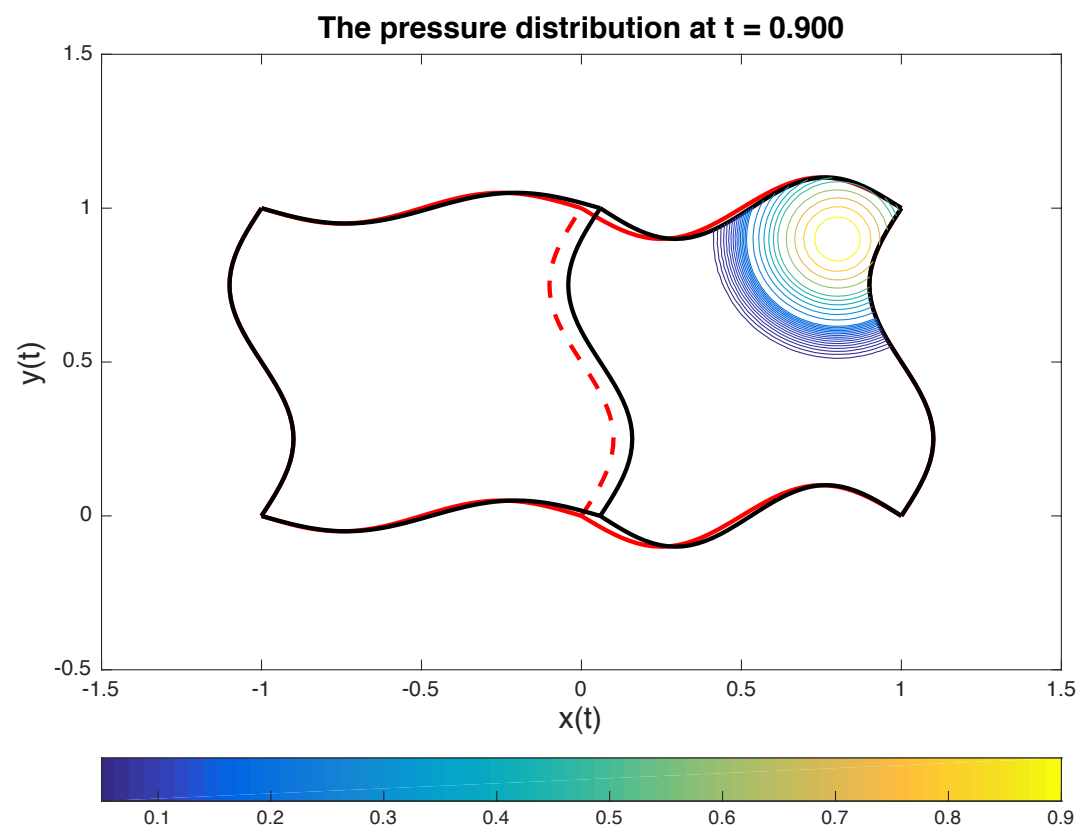

Figure 13: The pressure distribution.

where $E=[E x, E y]^{T}, H, J, \rho, \varepsilon$ and $\mu$ denote the electric field, the strength of the magnetic field, electric current density, charge density, permittivity and permeability. We prescribe $\rho=1, \varepsilon=1$ and $\mu=1$. By letting $J=0$, (48) is re-written in matrix-vector form as

$$
S U_{t}+A U_{x}+B U_{y}=0
$$

where $U=[H, E x, E y]^{T}$ and

$$
S=\left[\begin{array}{lll}
\mu & 0 & 0 \\
0 & \varepsilon & 0 \\
0 & 0 & \varepsilon
\end{array}\right], A=\left[\begin{array}{lll}
0 & 0 & 1 \\
0 & 0 & 0 \\
1 & 0 & 0
\end{array}\right], B=\left[\begin{array}{ccc}
0 & -1 & 0 \\
-1 & 0 & 0 \\
0 & 0 & 0
\end{array}\right]
$$

The matrices $A$ and $B$ in (49) are diagonalizable and have distinct eigenvalues. We decompose them as $A=X_{A} \Lambda_{A} X_{A}^{T}$ and $B=X_{B} \Lambda_{B} X_{B}^{T}$, where

$\Lambda_{A}=\Lambda_{B}=\left[\begin{array}{ccc}1 & 0 & 0 \\ 0 & 0 & 0 \\ 0 & 0 & -1\end{array}\right], X_{A}=\left[\begin{array}{ccc}-1 / \sqrt{2} & 0 & -1 / \sqrt{2} \\ 1 / \sqrt{2} & 0 & -1 / \sqrt{2} \\ 0 & 1 & 0\end{array}\right], X_{B}=\left[\begin{array}{ccc}1 / \sqrt{2} & 0 & 1 / \sqrt{2} \\ 0 & -1 & 0 \\ 1 / \sqrt{2} & 0 & -1 / \sqrt{2}\end{array}\right]$. 


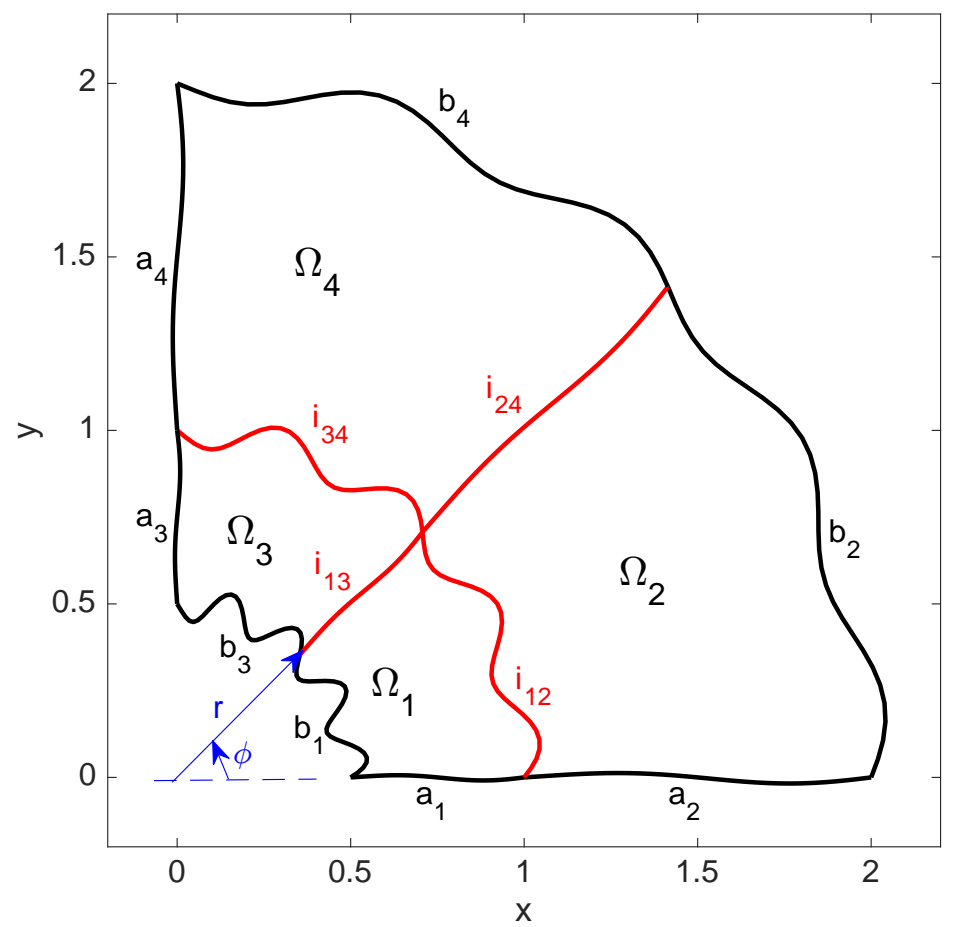

Figure 14: A schematic of four varying curved spatial domains and the interfaces at $t=0$.

Additionally, the characteristic variables become

$$
X_{A}^{T} U=\left[\frac{-H_{z}+E_{x}}{\sqrt{2}}, E_{y}, \frac{-H_{z}-E_{x}}{\sqrt{2}}\right]^{T}, X_{B}^{T} U=\left[\frac{H_{z}+E_{y}}{\sqrt{2}},-E_{x}, \frac{H_{z}-E_{y}}{\sqrt{2}}\right] .
$$

The matrix $S$ in (49) does not affect the well-posedness, conservation and stability analysis described in sections 2.2 and 3.2.

\subsubsection{An application}

We consider (49) posed on four curved time-dependent domains that are coupled through four interfaces as shown in Figure 14. The boundaries of the domains as well as the interfaces are varying as

$$
\Omega_{1}(t):\left\{\begin{array}{l}
r_{b_{1}}(t, \phi)=0.5-(0.2 /(2 \pi)) \sin (4 \pi t)+0.05 \sin \left(4 \pi \frac{\phi-\phi_{a_{1}}}{\phi_{i_{13}}-\phi_{a_{1}}}\right), \\
\phi_{a_{1}}(t, r)=-(0.5 /(2 \pi)) \sin (2 \pi t)+0.01 \sin \left(2 \pi \frac{r-r_{b_{1}}}{r_{i_{12}}-r_{b_{1}}}\right), \\
r_{i_{12}}(t, \phi)=1-(0.5 /(2 \pi)) \sin (2 \pi t)+0.05 \sin \left(4 \pi \frac{\phi-\phi_{a_{1}}}{\phi_{i_{13}}-\phi_{a_{1}}}\right), \\
\phi_{i_{13}}(t, r)=(\pi / 4)+(0.5 /(2 \pi)) \sin (2 \pi t)+0.01 \sin \left(2 \pi \frac{r-r_{b_{1}}}{r_{i_{12}}-r_{b_{1}}}\right),
\end{array}\right.
$$




$$
\begin{aligned}
& \left(r_{b_{2}}(t, \phi)=1-(0.5 /(2 \pi)) \sin (2 \pi t)+0.05 \sin \left(4 \pi \frac{\phi-\phi_{a_{2}}}{\phi_{i_{24}}-\phi_{a_{2}}}\right)\right. \\
& \phi_{a_{2}}(t, r)=-(0.5 /(2 \pi)) \sin (2 \pi t)+0.01 \sin \left(2 \pi \frac{r-r_{i_{12}}}{r_{b_{2}}-r_{i_{12}}}\right), \\
& r_{i_{12}}(t, \phi)=1-(0.5 /(2 \pi)) \sin (2 \pi t)+0.05 \sin \left(4 \pi \frac{\phi-\phi_{a_{2}}}{\phi_{i_{24}}-\phi_{a_{2}}}\right), \\
& \phi_{i_{24}}(t, r)=(\pi / 4)+(0.5 /(2 \pi)) \sin (2 \pi t)+0.01 \sin \left(2 \pi \frac{r-r_{i_{12}}}{r_{b_{2}}-r_{i_{12}}}\right), \\
& \Omega_{3}(t):\left\{\begin{array}{l}
r_{b_{3}}(t, \phi)=0.5-(0.2 /(2 \pi)) \sin (4 \pi t)+0.05 \sin \left(4 \pi \frac{\phi-\phi_{i_{13}}}{\phi_{a_{3}}-\phi_{i_{13}}}\right), \\
\phi_{a_{3}}(t, r)=(\pi / 2)+(0.2 /(2 \pi)) \sin (4 \pi t)+0.01 \sin \left(2 \pi \frac{r-r_{b_{3}}}{r_{i_{34}}-r_{b_{3}}}\right), \\
r_{i_{34}}(t, \phi)=1-(0.5 /(2 \pi)) \sin (2 \pi t)+0.05 \sin \left(4 \pi \frac{\phi-\phi_{i_{13}}}{\phi_{a_{3}}-\phi_{i_{13}}}\right), \\
\phi_{i_{34}}(t, r)=(\pi / 4)+(0.5 /(2 \pi)) \sin (2 \pi t)+0.01 \sin \left(2 \pi \frac{r-r_{b_{3}}}{r_{i_{34}}-r_{b_{3}}}\right),
\end{array}\right.
\end{aligned}
$$

and

$$
\Omega_{4}(t):\left\{\begin{array}{l}
r_{b_{4}}(t, \phi)=1-(0.5 /(2 \pi)) \sin (2 \pi t)+0.05 \sin \left(4 \pi \frac{\phi-\phi_{i_{24}}}{\phi_{a_{4}}-\phi_{i_{24}}}\right), \\
\phi_{a_{4}}(t, r)=(\pi / 2)+(0.2 /(2 \pi)) \sin (4 \pi t)+0.01 \sin \left(2 \pi \frac{r-r_{i_{34}}}{r_{b_{4}}-r_{i_{34}}}\right), \\
r_{i_{34}}(t, \phi)=1-(0.5 /(2 \pi)) \sin (2 \pi t)+0.05 \sin \left(4 \pi \frac{\phi-\phi_{i_{24}}}{\phi_{a_{4}}-\phi_{i_{24}}}\right), \\
\phi_{i_{24}}(t, r)=(\pi / 4)+(0.5 /(2 \pi)) \sin (2 \pi t)+0.01 \sin \left(2 \pi \frac{r-r_{i_{34}}}{r_{b_{4}}-r_{i_{34}}}\right) .
\end{array}\right.
$$

The boundaries and interfaces given in (53)-(56) are shown graphically in Figure 14. We transform the domains $\Omega_{1,2,3,4}$ from Cartesian coordinates into curvilinear coordinates, $\xi, \eta$ by using time-dependent coordinate transformations. A schematic of the corresponding transformed domains, and fixed interfaces are shown in Figure 15. 


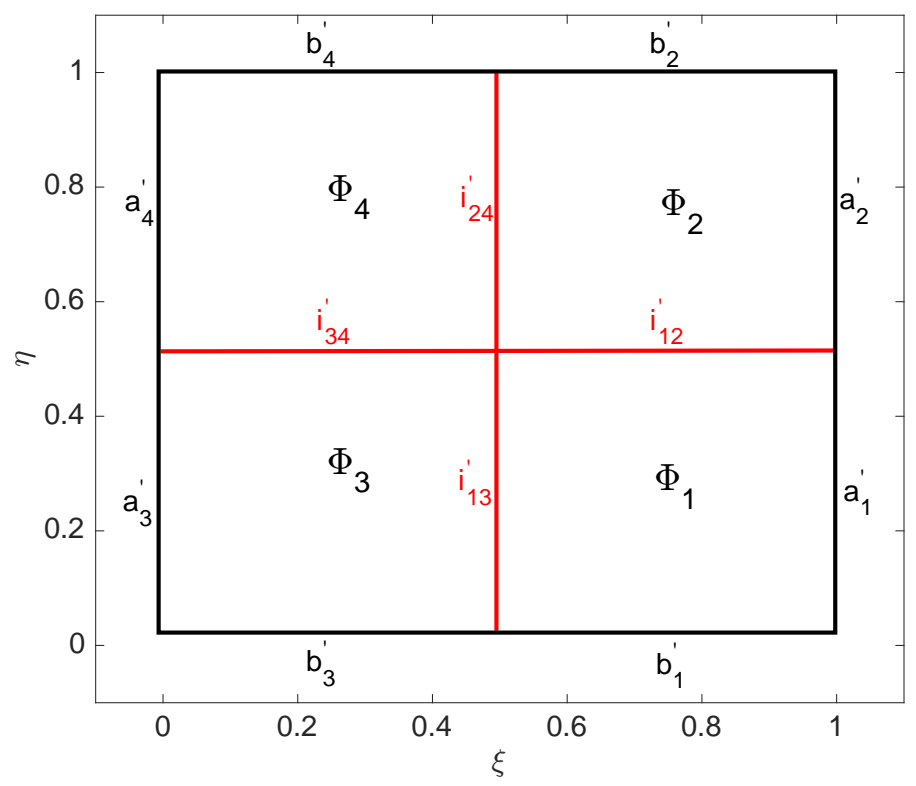

Figure 15: A schematic of the fixed domains and the interfaces.

We consider two magnetic sources, $H_{1}(t, x, y)$ and $H_{2}(t, x, y)$ located at $(x, y)=$ $(1.4,0.5)$ and $(x, y)=(0.5,1.4)$, respectively, where

$$
\begin{aligned}
& H_{1}(t, x, y)=0.1 \exp \left(-10\left[(t-0.2)^{2}+(x-1.4)^{2}+(y-0.5)^{2}\right]\right), \\
& H_{2}(t, x, y)=0.1 \exp \left(-10\left[(t-0.5)^{2}+(x-0.5)^{2}+(y-1.4)^{2}\right]\right) .
\end{aligned}
$$

The magnetic sources are injected into (49) as a forcing function. Characteristic boundary conditions with magnetic data taken from (57) and zero data for the electric field are used.

A spatial mesh of size $41 \times 41$ in each sub-domain and 81 time levels for $t \in[0,1]$ are constructed. A third order accurate SBP operator in space together with a fifth order accurate SBP operator in time is used.

The strength of the magnetic sources and the corresponding induced electric field at different times are shown in Figures 16-39. To visualize the domain variations, the initial domains, $\Omega_{1,2,3,4}(0)$, are shown with dashed curves for reference.

As seen in Figures 16-39, by changing the strength of the magnetic sources, an electric field is induced in the domain. Moreover, decreasing and increasing the strength of the magnetic sources result in a clock-wise and counter clock-wise electric field around the sources, respectively. 


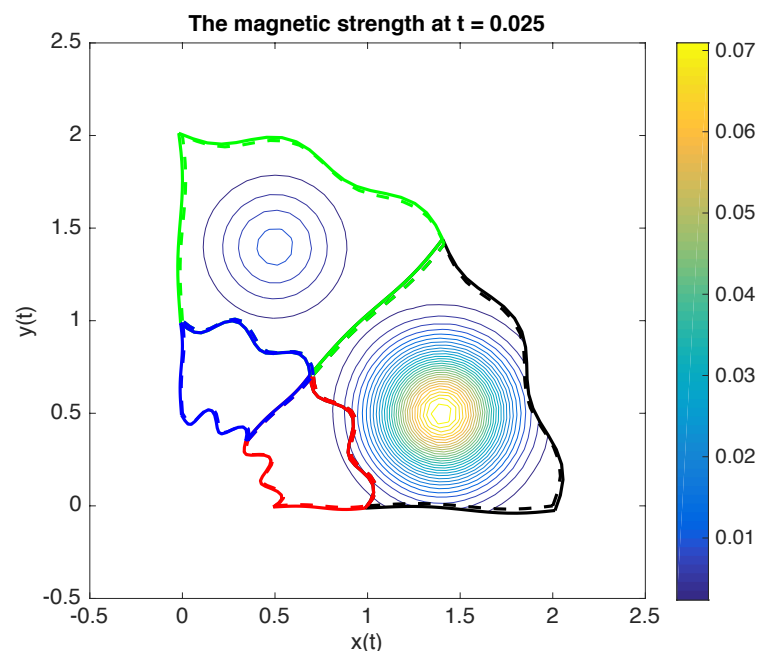

Figure 16: The magnetic sources.

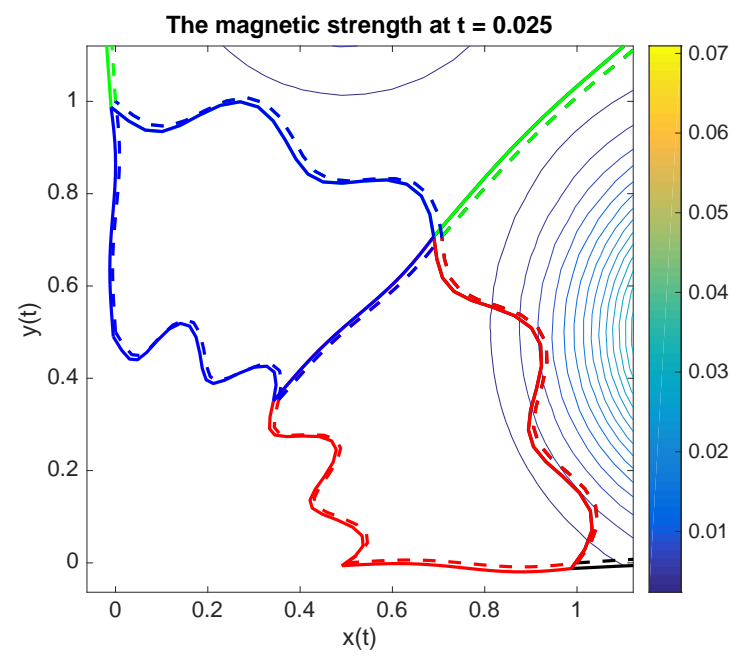

Figure 18: A blow-up of the magnetic sources.

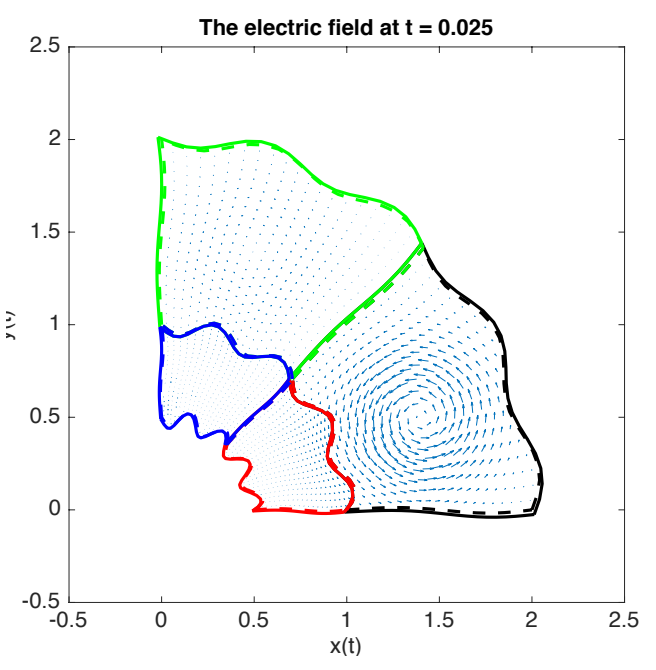

Figure 17: The induced electric field.

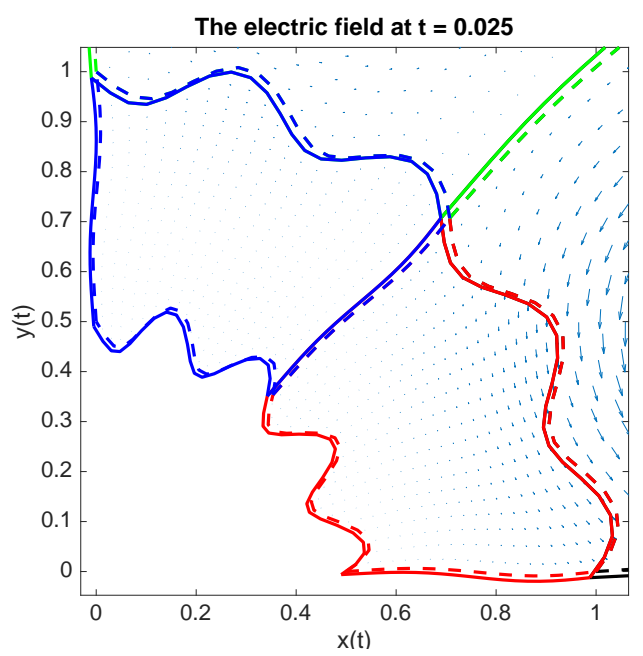

Figure 19: A blow-up of the induced electric field. 


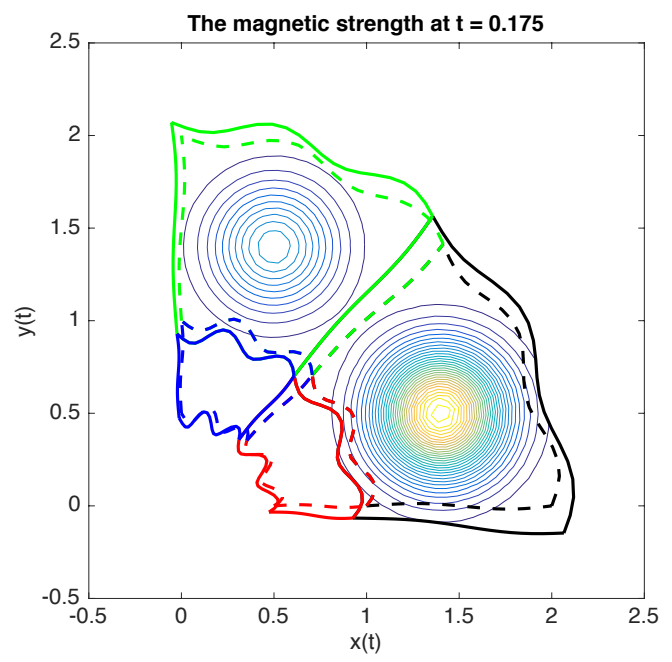

Figure 20: The magnetic sources.

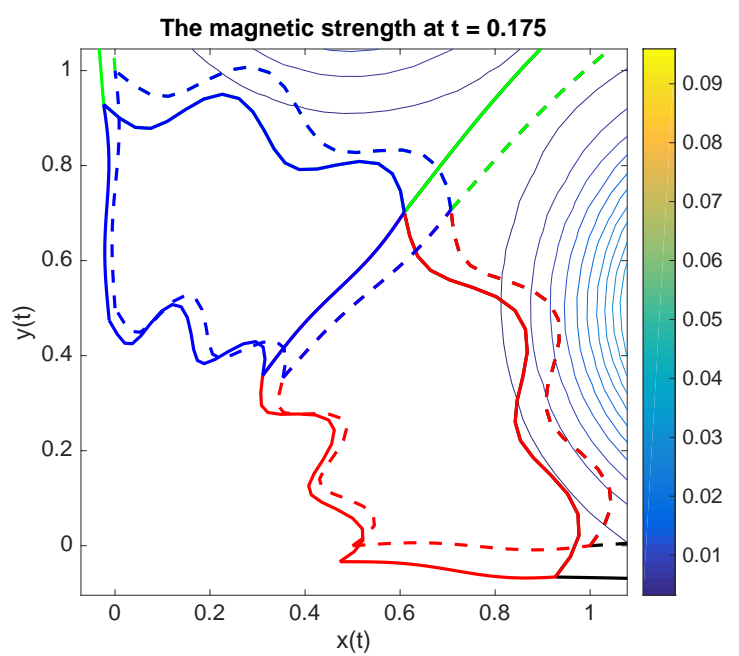

Figure 22: A blow-up of the magnetic sources.

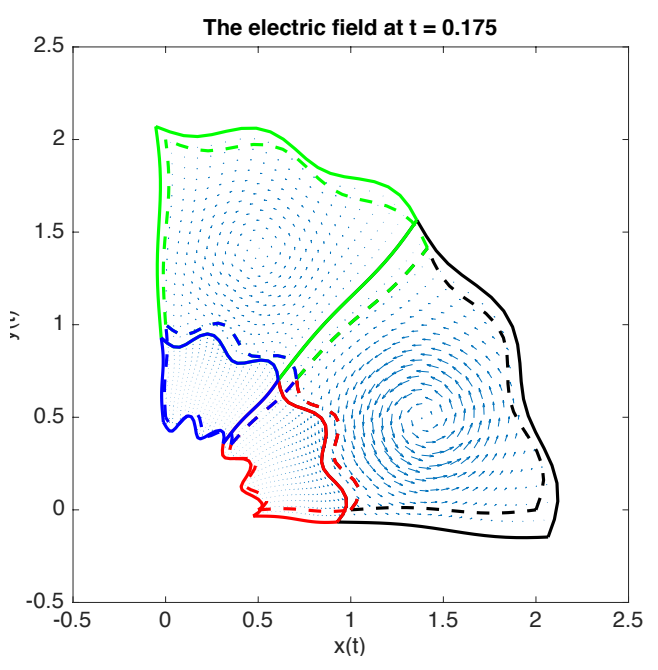

Figure 21: The induced electric field.

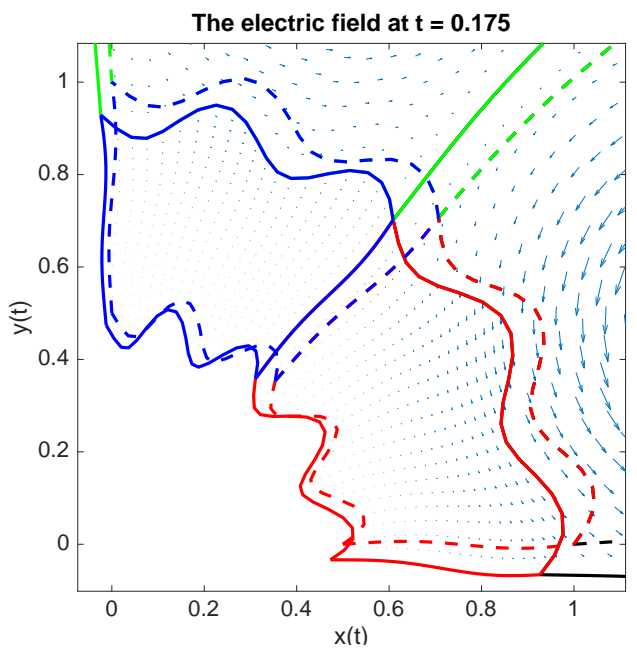

Figure 23: A blow-up of the induced electric field. 


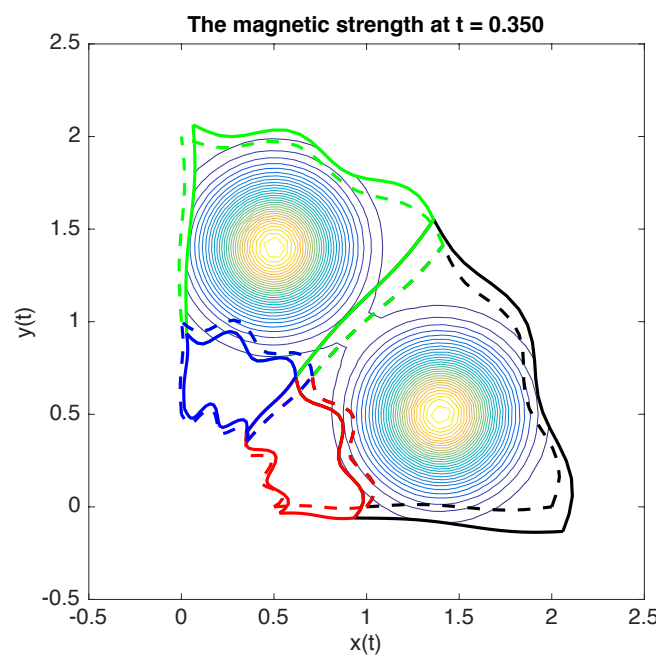

Figure 24: The magnetic sources.

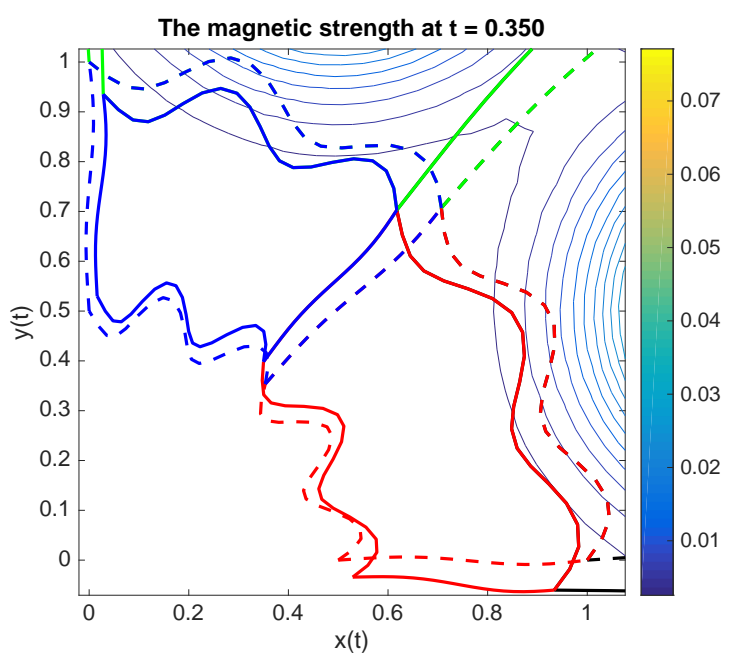

Figure 26: A blow-up of the magnetic sources.
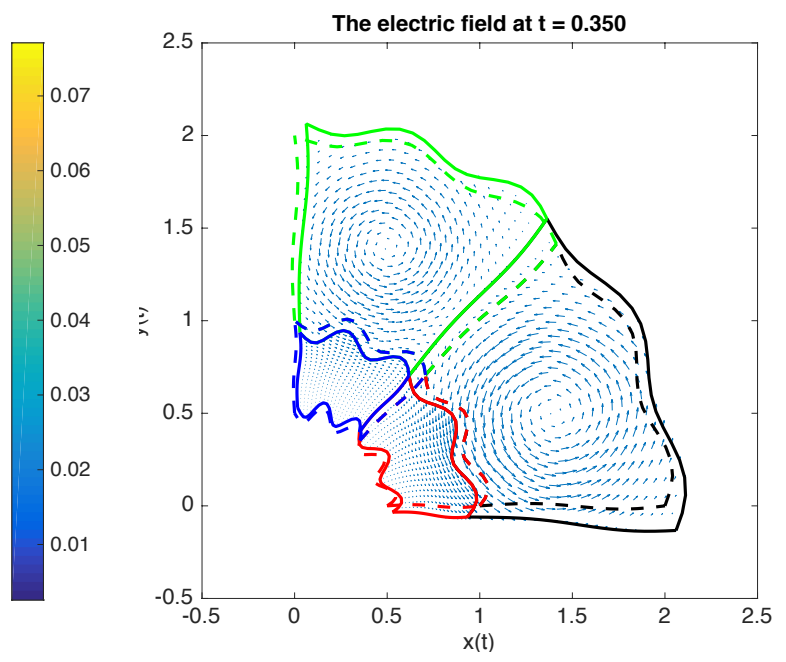

Figure 25: The induced electric field.

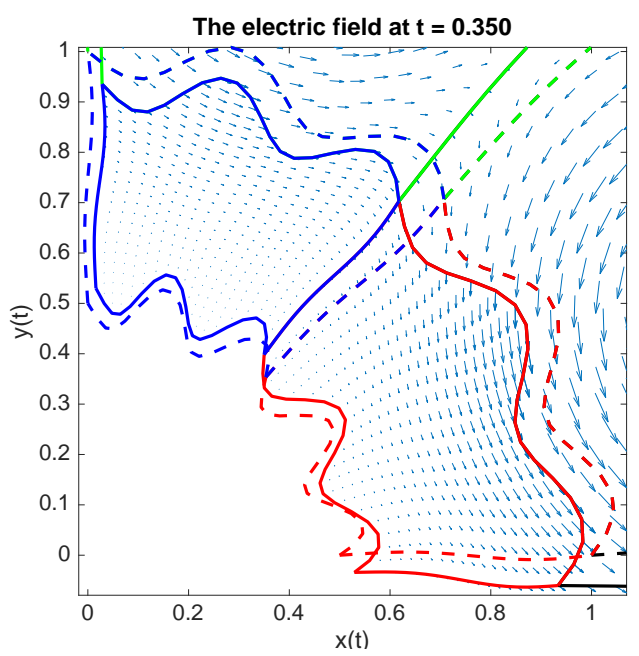

Figure 27: A blow-up of the induced electric field. 


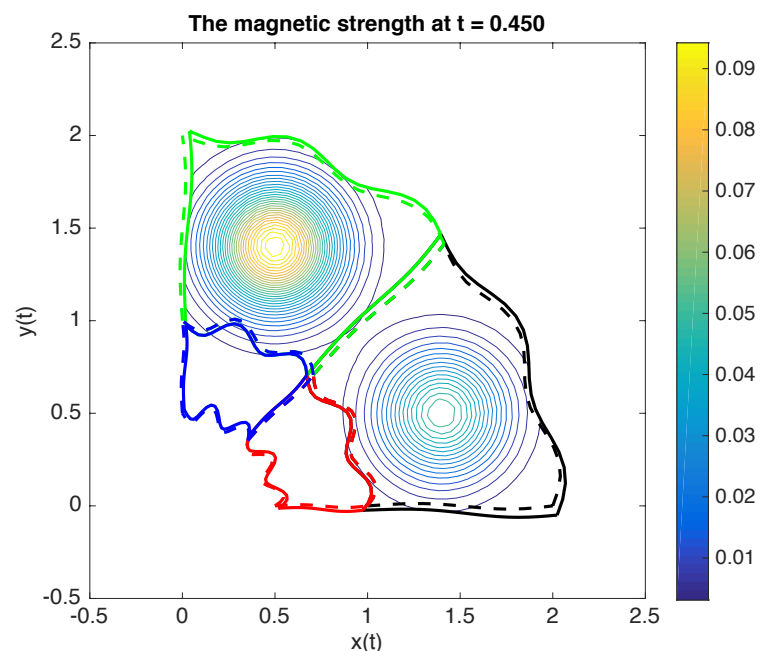

Figure 28: The magnetic sources.

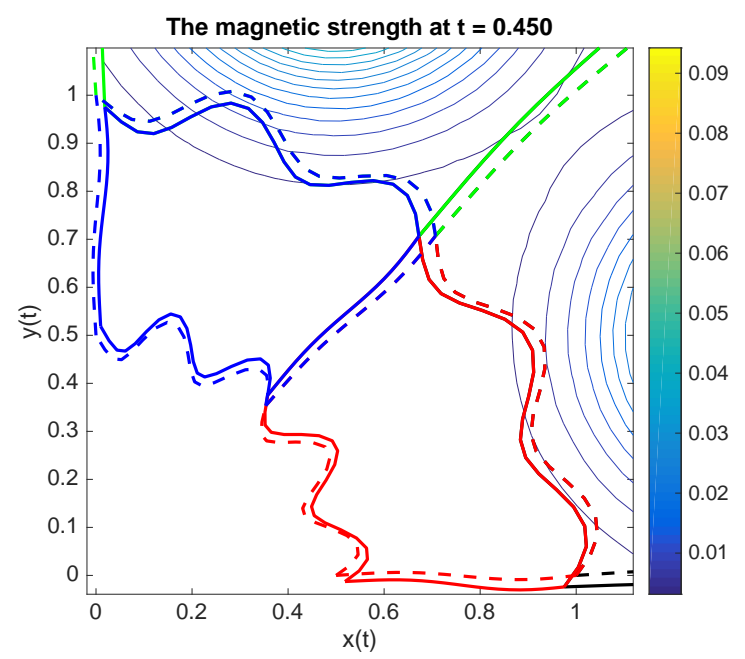

Figure 30: A blow-up of the magnetic sources.

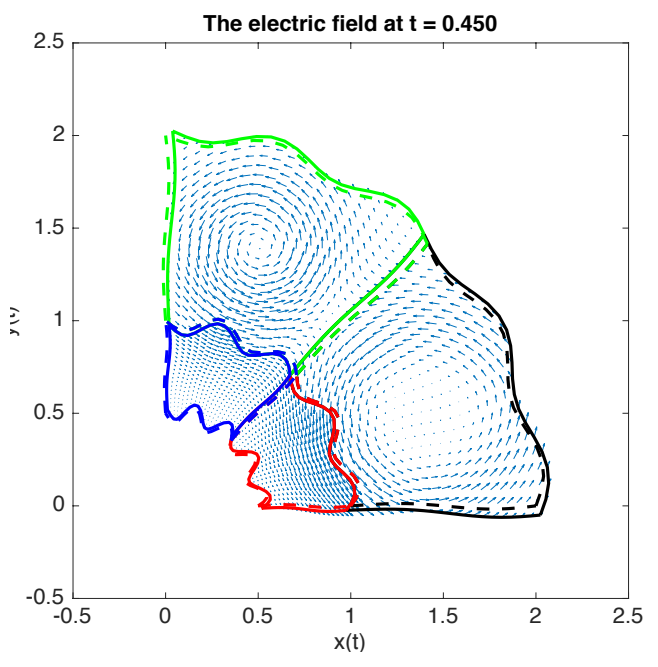

Figure 29: The induced electric field.

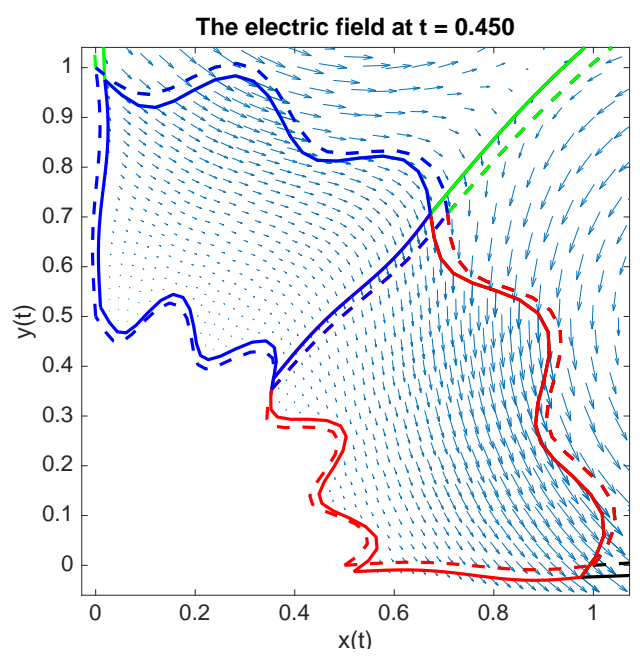

Figure 31: A blow-up of the induced electric field. 


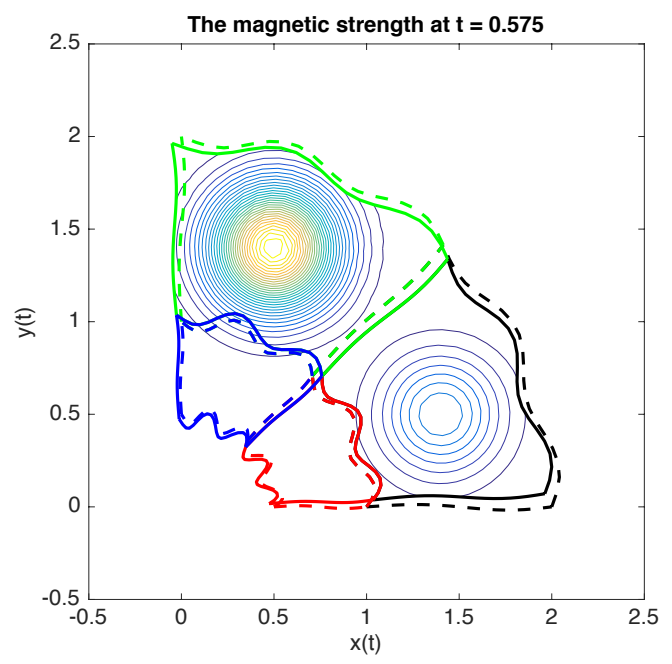

Figure 32: The magnetic sources.

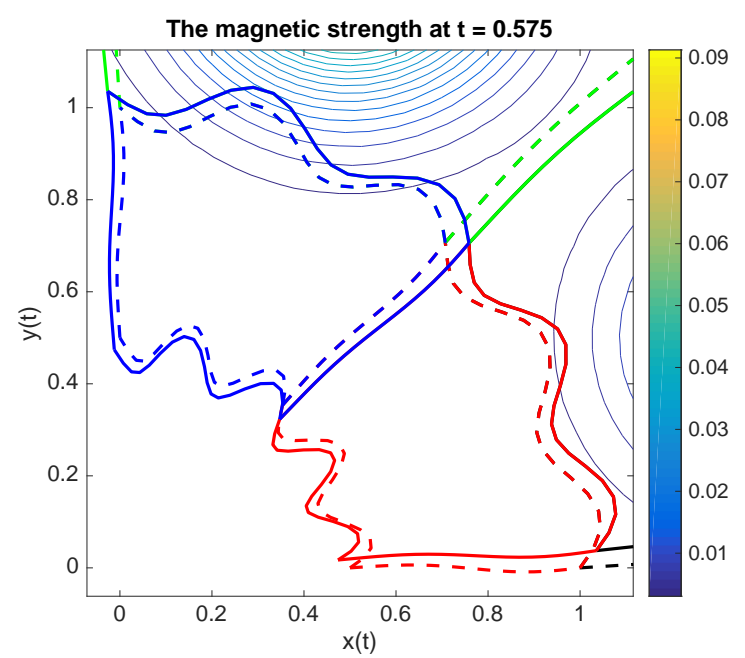

Figure 34: A blow-up of the magnetic sources.

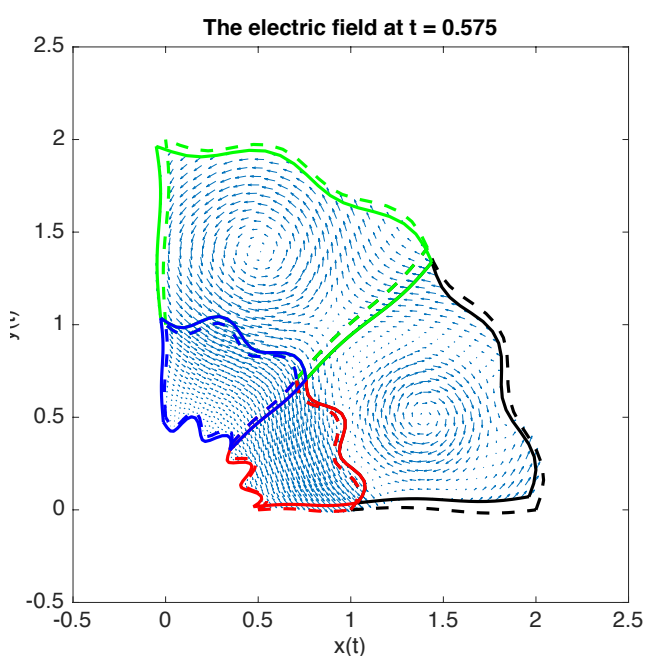

Figure 33: The induced electric field.

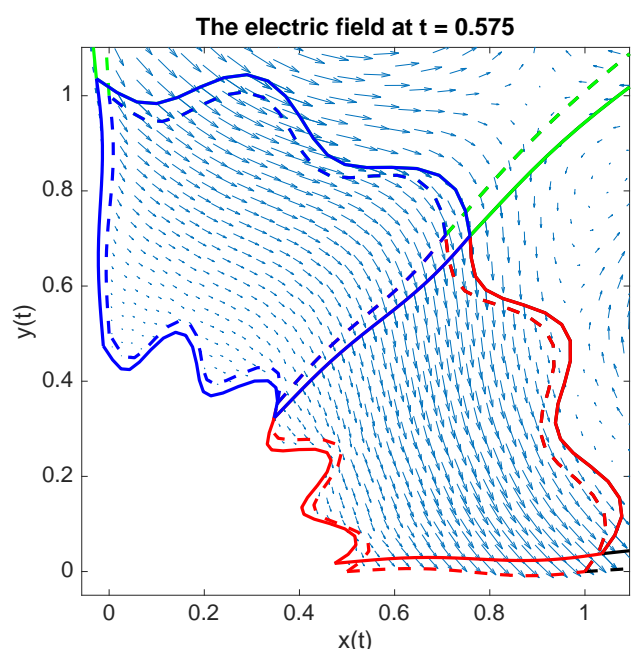

Figure 35: A blow-up of the induced electric field. 


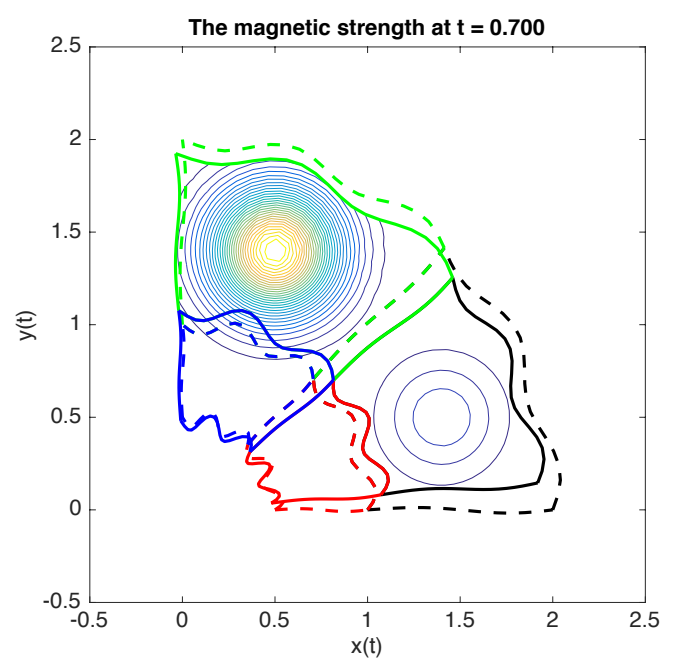

Figure 36: The magnetic sources.

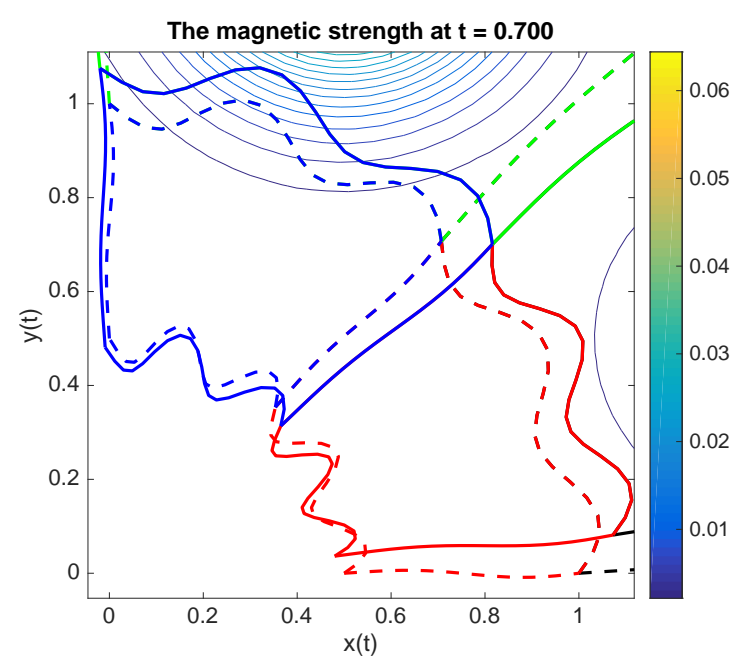

Figure 38: A blow-up of the magnetic sources.
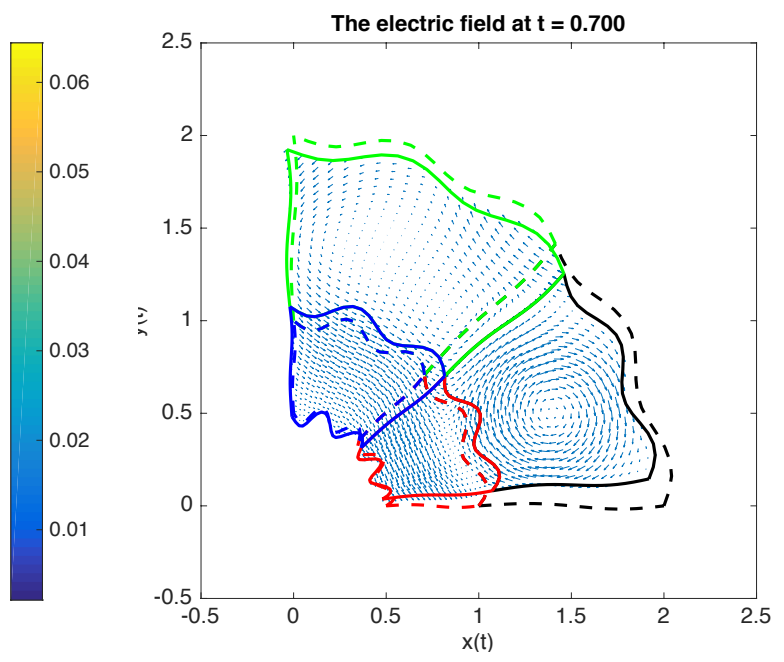

Figure 37: The induced electric field.

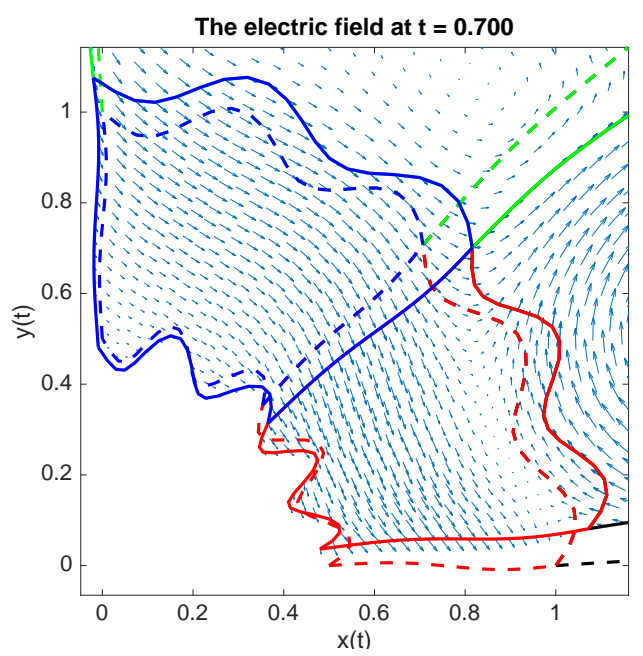

Figure 39: A blow-up of the induced electric field. 


\section{Summary and conclusions}

We have constructed stable and conservative numerical schemes on summationby-parts form for multi-domain problems with time-dependent deforming interfaces.

We have derived conditions for discrete conservation and stability such that the scheme approximates the continuous problem. The interface procedure was constructed in such a way that a dissipative, conservative and stable procedure is obtained automatically with respect to the movements and deformations of the interface.

The correct rates of convergence toward the exact solution, were shown. Applications using the linearized Euler equations and the Maxwell equations posed on time-dependent multi-block geometries with moving and deforming interfaces between the blocks were used to illustrate the performance of the procedure.

\section{Appendices}

\section{A. Appendix}

First, we multiply the first and second system of equations in (9) with $\phi_{L, R}(\tau, \xi, \eta)$ and integrate in space and time to obtain

$$
\begin{aligned}
& \int_{0}^{T} \iint_{\Phi_{L}}\left[\phi_{L}^{T}\left(J_{L} U\right)_{\tau}+\phi_{L}^{T}\left(A_{L} U\right) \xi+\phi_{L}^{T}\left(B_{L} U\right)_{\eta}\right] d \Phi d \tau=0, \\
& \int_{0}^{T} \iint_{\Phi_{R}}\left[\phi_{R}^{T}\left(J_{R} V\right)_{\tau}+\phi_{R}^{T}\left(A_{R} V\right) \xi+\phi_{R}^{T}\left(B_{R} V\right)_{\eta}\right] d \Phi d \tau=0 .
\end{aligned}
$$

Adding and subtracting the terms $\left(\phi_{L}^{T}\right)_{\tau}\left(J_{L} U\right)+\left(\phi_{L}^{T}\right)_{\xi}\left(A_{L} U\right)+\left(\phi_{L}^{T}\right)_{\eta}\left(B_{L} U\right)$ and $\left(\phi_{R}^{T}\right)_{\tau}\left(J_{R} V\right)+\left(\phi_{R}^{T}\right)_{\xi}\left(A_{R} V\right)+\left(\phi_{R}^{T}\right)_{\eta}\left(B_{R} V\right)$ to the first and second integral argu- 
ments in (58), lead to

$$
\begin{gathered}
\int_{0}^{T} \iint_{\Phi_{L}}\left[\left(\phi_{L}^{T} J_{L} U\right)_{\tau}+\left(\phi_{L}^{T} A_{L} U\right)_{\xi}+\left(\phi_{L}^{T} B_{L} U\right)_{\eta}\right] d \Phi d \tau= \\
\int_{0}^{T} \iint_{\Phi_{L}}\left[\left(\phi_{L}^{T}\right)_{\tau} J_{L} U+\left(\phi_{L}^{T}\right)_{\xi} A_{L} U+\left(\phi_{L}^{T}\right)_{\eta} B_{L} U\right] d \Phi d \tau, \\
\int_{0}^{T} \iint_{\Phi_{R}}\left[\left(\phi_{R}^{T} J_{R} V\right)_{\tau}+\left(\phi_{R}^{T} A_{R} V\right)_{\xi}+\left(\phi_{R}^{T} B_{R} V\right)_{\eta}\right] d \Phi d \tau= \\
\int_{0}^{T} \iint_{\Phi_{R}}\left[\left(\phi_{R}^{T}\right)_{\tau} J_{R} V+\left(\phi_{R}^{T}\right)_{\xi} A_{R} V+\left(\phi_{R}^{T}\right)_{\eta} B_{R} V\right] d \Phi d \tau .
\end{gathered}
$$

Now, we integrate the first arguments on the left hand sides of both equations in (59) in time, and use Green-Gauss theorem for the second and third arguments and obtain

$$
\begin{aligned}
\left.\iint_{\Phi_{L}}\left(\phi_{L}^{T} J_{L} U\right)\right|_{\tau=0} ^{\tau=T} d \Phi & +\int_{0}^{T} \oint_{\delta \Phi_{L}}\left[\phi_{L}^{T}\left(A_{L}, B_{L}\right) \cdot n_{L} U\right] d \Phi d \tau \\
& =\int_{0}^{T} \iint_{\Phi_{L}}\left[\left(\phi_{L}^{T}\right)_{\tau} J_{L} U+\left(\phi_{L}^{T}\right)_{\xi} A_{L} U+\left(\phi_{L}^{T}\right)_{\eta} B_{L} U\right] d \Phi d \tau \\
\left.\iint_{\Phi_{R}}\left(\phi_{R}^{T} J_{R} V\right)\right|_{\tau=0} ^{\tau=T} d \Phi & +\int_{0}^{T} \oint_{\delta \Phi_{R}}\left[\phi_{R}^{T}\left(A_{R}, B_{R}\right) \cdot n_{R} V\right] d \Phi d \tau \\
& =\int_{0}^{T} \iint_{\Phi_{R}}\left[\left(\phi_{R}^{T}\right)_{\tau} J_{R} V+\left(\phi_{R}^{T}\right)_{\xi} A_{R} V+\left(\phi_{R}^{T}\right)_{\eta} B_{R} V\right] d \Phi d \tau .
\end{aligned}
$$

Since $\phi_{L}$ and $\phi_{R}$ vanish at the far-field spatial boundaries and also temporal borders, all the terms on the left hand side of the equality in (60), except the interface terms, vanish. The result is

$$
\begin{gathered}
\int_{0}^{T} \int \phi_{L}^{T} A_{L} U d \eta d \tau=\int_{0}^{T} \iint_{\Phi_{L}}\left[\left(\phi_{L}^{T}\right)_{\tau} J_{L} U+\left(\phi_{L}^{T}\right)_{\xi} A_{L} U+\left(\phi_{L}^{T}\right)_{\eta} B_{L} U\right] d \Phi d \tau, \\
-\int_{0}^{T} \int \phi_{R}^{T} A_{R} V d \eta d \tau=\int_{0}^{T} \iint_{\Phi_{R}}\left[\left(\phi_{R}^{T}\right)_{\tau} J_{R} V+\left(\phi_{R}^{T}\right)_{\xi} A_{R} V+\left(\phi_{R}^{T}\right)_{\eta} B_{R} V\right] d \Phi d \tau .
\end{gathered}
$$

We add the zero terms $\phi_{L}^{T}\left[\left(J_{L}\right)_{\tau}+\left(A_{L}\right)_{\xi}+\left(B_{L}\right)_{\eta}\right] U$ and $\phi_{R}^{T}\left[\left(J_{R}\right)_{\tau}+\left(A_{R}\right)_{\xi}+\left(B_{R}\right)_{\eta}\right] V$, see (8), to the right hand sides of the equalities in first and second equation in (61), 
respectively. By using the symmetry properties of the matrices one can arrive at

$$
\begin{aligned}
& \int_{0}^{T} \iint_{\Phi_{L}}\left[U^{T}\left[\left(J_{L} \phi_{L}\right)_{\tau}+\left(A_{L} \phi_{L}\right)_{\xi}+\left(B_{L} \phi_{L}\right) \eta\right]\right]^{T} d \Phi d \tau=\int_{0}^{T} \int \phi_{L}^{T} A_{L} U d \eta d \tau \\
& \int_{0}^{T} \iint_{\Phi_{R}}\left[V^{T}\left[\left(J_{R} \phi_{R}\right)_{\tau}+\left(A_{R} \phi_{R}\right)_{\xi}+\left(B_{R} \phi_{R}\right)_{\eta}\right]^{T} d \Phi d \tau=-\int_{0}^{T} \int \phi_{R}^{T} A_{R} V d \eta d \tau .\right.
\end{aligned}
$$

Adding the results of the left and right problem and considering $\phi_{L}=\phi_{R}:=\phi_{I}$ at the interface, give

$$
\begin{aligned}
& \int_{0}^{T} \iint_{\Phi_{L}}\left[U^{T}\left[\left(J_{L} \phi_{L}\right)_{\tau}+\left(A_{L} \phi_{L}\right)_{\xi}+\left(B_{L} \phi_{L}\right)_{\eta}\right]\right]^{T} d \Phi d \tau+ \\
& \int_{0}^{T} \iint_{\Phi_{R}}\left[V^{T}\left[\left(J_{R} \phi_{R}\right)_{\tau}+\left(A_{R} \phi_{R}\right)_{\xi}+\left(B_{R} \phi_{R}\right)_{\eta}\right]\right]^{T} d \Phi d \tau=\int_{0}^{T} \int_{I} \underbrace{\phi_{I}^{T}\left(A_{L_{I}} U_{I}-A_{R_{I}} V_{I}\right)}_{I T} d \eta d \tau .
\end{aligned}
$$

\section{B. Appendix}

First, we multiply (21) from the left with $\phi_{L}^{T} \mathcal{P}_{L}$ and $\phi_{R}^{T} \mathcal{P}_{R}$, where $\mathcal{P}_{L, R}=$ $P_{\tau} \otimes\left(P_{\xi}\right)_{L, R} \otimes P_{\eta} \otimes I$ and use the SBP property (18) to obtain

$$
\begin{aligned}
& \frac{1}{2} \phi_{L}^{T}\left[\left(-Q_{\tau}^{T} \otimes P_{\xi} \otimes P_{\eta} \otimes I\right) \mathbf{J}_{L}+\mathbf{J}_{L}\left(-Q_{\tau}^{T} \otimes P_{\xi} \otimes P_{\eta} \otimes I\right)+P_{L}\left(\mathbf{J}_{L}\right)_{\tau}\right] \mathbf{U}+ \\
& \frac{1}{2} \phi_{L}^{T}\left[\left(P_{\tau} \otimes-Q_{\xi}^{T} \otimes P_{\eta} \otimes I\right) \mathbf{A}_{L}+\mathbf{A}_{L}\left(P_{\tau}^{T} \otimes-Q_{\xi}^{T} \otimes P_{\eta} \otimes I\right)+P_{L}\left(\mathbf{A}_{L}\right) \xi\right] \mathbf{U}+ \\
& \frac{1}{2} \phi_{L}^{T}\left[\left(P_{\tau} \otimes P_{\xi} \otimes-Q_{\eta}^{T} \otimes I\right) \mathbf{B}_{L}+\mathbf{B}_{L}\left(P_{\tau} \otimes P_{\xi} \otimes-Q_{\eta}^{T} \otimes I\right)+P_{L}\left(\mathbf{B}_{L}\right)_{\eta}\right] \mathbf{U}= \\
& -\phi_{L}^{T}\left(P_{\tau} \otimes E_{1} \otimes P_{\eta} \otimes I\right) \mathbf{A}_{L} \mathbf{U}+\phi_{L}^{T}\left(P_{\tau} \otimes E_{1} \otimes P_{\eta} \otimes I\right) \Sigma_{L}(\mathbf{U}-\mathbf{V}),
\end{aligned}
$$

$$
\begin{aligned}
& \frac{1}{2} \phi_{R}^{T}\left[\left(-Q_{\tau}^{T} \otimes P_{\xi} \otimes P_{\eta} \otimes I\right) \mathbf{J}_{R}+\mathbf{J}_{R}\left(-Q_{\tau}^{T} \otimes P_{\xi} \otimes P_{\eta} \otimes I\right)+P_{R}\left(\mathbf{J}_{R}\right)_{\tau}\right] \mathbf{V}+ \\
& \frac{1}{2} \phi_{R}^{T}\left[\left(P_{\tau} \otimes-Q_{\xi}^{T} \otimes P_{\eta} \otimes I\right) \mathbf{A}_{R}+\mathbf{A}_{R}\left(P_{\tau}^{T} \otimes-Q_{\xi}^{T} \otimes P_{\eta} \otimes I\right)+P_{R}\left(\mathbf{A}_{R}\right)_{\xi}\right] \mathbf{V}+ \\
& \frac{1}{2} \phi_{R}^{T}\left[\left(P_{\tau} \otimes P_{\xi} \otimes-Q_{\eta}^{T} \otimes I\right) \mathbf{B}_{R}+\mathbf{B}_{R}\left(P_{\tau} \otimes P_{\xi} \otimes-Q_{\eta}^{T} \otimes I\right)+P_{R}\left(\mathbf{B}_{R}\right) \eta\right] \mathbf{V}= \\
& \phi_{R}^{T}\left(P_{\tau} \otimes E_{0} \otimes P_{\eta} \otimes I\right) \mathbf{A}_{R} \mathbf{V}+\phi_{R}^{T}\left(P_{\tau} \otimes E_{0} \otimes P_{\eta} \otimes I\right) \Sigma_{R}(\mathbf{V}-\mathbf{U}) .
\end{aligned}
$$


Equation (64) can be re-written as

$$
\begin{aligned}
& -\frac{1}{2}\left[\left[\left(D_{\tau}\right)_{L} \phi_{L}\right]^{T} P_{L} \mathbf{J}_{L}+\left[\left(D_{\tau}\right)_{L} \mathbf{J}_{L} \phi_{L}\right]^{T} P_{L}-\phi_{L}^{T} P_{L}\left(\mathbf{J}_{L}\right)_{\tau}\right] \mathbf{U}+ \\
& \left.-\frac{1}{2}\left[\left(D_{\xi}\right)_{L} \phi_{L}\right]^{T} P_{L} \mathbf{A}_{L}+\left[\left(D_{\xi}\right)_{L} \mathbf{A}_{L} \phi_{L}\right]^{T} P_{L}-\phi_{L}^{T} P_{L}\left(\mathbf{A}_{L}\right) \xi\right] \mathbf{U}+ \\
& -\frac{1}{2}\left[\left[\left(D_{\eta}\right)_{L} \phi_{L}\right]^{T} P_{L} \mathbf{B}_{L}+\left[\left(D_{\eta}\right)_{L} \mathbf{B}_{L} \phi_{L}\right]^{T} P_{L}-\phi_{L}^{T} P_{L}\left(\mathbf{B}_{L}\right)_{\eta}\right] \mathbf{U}= \\
& -\phi_{L}^{T}\left(P_{\tau} \otimes E_{1} \otimes P_{\eta} \otimes I\right) \mathbf{A}_{L} \mathbf{U}+\phi_{L}^{T}\left(P_{\tau} \otimes E_{1} \otimes P_{\eta} \otimes I\right) \Sigma_{L}(\mathbf{U}-\mathbf{V}), \\
& -\frac{1}{2}\left[\left[\left(D_{\tau}\right)_{R} \phi_{R}\right]^{T} P_{R} \mathbf{J}_{R}+\left[\left(D_{\tau}\right)_{R} \mathbf{J}_{R} \phi_{R}\right]^{T} P_{R}-\phi_{R}^{T} P_{R}\left(\mathbf{J}_{R}\right)_{\tau}\right] \mathbf{V}+ \\
& \left.-\frac{1}{2}\left[\left(D_{\xi}\right)_{R} \phi_{R}\right]^{T} P_{R} \mathbf{A}_{R}+\left[\left(D_{\xi}\right)_{R} \mathbf{A}_{R} \phi_{R}\right]^{T} P_{R}-\Phi_{R}^{T} P_{R}\left(\mathbf{A}_{R}\right) \xi\right] \mathbf{V}+ \\
& -\frac{1}{2}\left[\left[\left(D_{\eta}\right)_{R} \phi_{R}\right]^{T} P_{R} \mathbf{B}_{R}+\left[\left(D_{\eta}\right)_{R} \mathbf{B}_{R} \phi_{R}\right]^{T} P_{R}-\phi_{R}^{T} P_{R}\left(\mathbf{B}_{R}\right)_{\eta}\right] \mathbf{V}= \\
& \phi_{R}^{T}\left(P_{\tau} \otimes E_{0} \otimes P_{\eta} \otimes I\right) \mathbf{A}_{R} \mathbf{V}+\phi_{R}^{T}\left(P_{\tau} \otimes E_{0} \otimes P_{\eta} \otimes I\right) \Sigma_{R}(\mathbf{V}-\mathbf{U}) .
\end{aligned}
$$

By using the symmetry property of the matrices and the fact that $P_{L, R}$ commute with the block diagonal matrices $\mathbf{A}_{L, R}$ and $\mathbf{B}_{L, R}$, we arrive at

$$
\begin{aligned}
& \frac{1}{2}\left[\mathbf{U}^{T} P_{L}\left[\mathbf{J}_{L}\left(D_{\tau}\right)_{L} \phi_{L}+\left(D_{\tau}\right)_{L} \mathbf{J}_{L} \phi_{L}-\left(\mathbf{J}_{L}\right)_{\tau} \phi_{L}\right]\right]^{T}+ \\
& \frac{1}{2}\left[\mathbf{U}^{T} P_{L}\left[\left(\mathbf{A}_{L}\left(D_{\xi}\right)_{L} \phi_{L}+\left(D_{\xi}\right)_{L} \mathbf{A}_{L} \phi_{L}-\left(\mathbf{A}_{L}\right)_{\xi} \Phi_{L}\right]\right]^{T}+\right. \\
& \frac{1}{2}\left[\mathbf{U}^{T} P_{L}\left[\left(\mathbf{B}_{L}\left(D_{\eta}\right)_{L} \phi_{L}+\left(D_{\eta}\right)_{L} \mathbf{B}_{L} \phi_{L}-\left(\mathbf{B}_{L}\right)_{\eta} \Phi_{L}\right]\right]^{T}=\right. \\
& \phi_{L}^{T}\left(P_{\tau} \otimes E_{1} \otimes P_{\eta} \otimes I\right) \mathbf{A}_{L} \mathbf{U}-\phi_{L}^{T}\left(P_{\tau} \otimes E_{1} \otimes P_{\eta} \otimes I\right) \Sigma_{L}(\mathbf{U}-\mathbf{V}), \\
& \frac{1}{2}\left[\mathbf{V}^{T} P_{R}\left[\mathbf{J}_{R}\left(D_{\tau}\right)_{R} \phi_{R}+\left(D_{\tau}\right)_{R} \mathbf{J}_{R} \phi_{R}-\left(\mathbf{J}_{R}\right)_{\tau} \phi_{R}\right]\right]^{T}+ \\
& \frac{1}{2}\left[\mathbf{V}^{T} P_{R}\left[\left(\mathbf{A}_{R}\left(D_{\xi}\right)_{R} \phi_{R}+\left(D_{\xi}\right)_{R} \mathbf{A}_{R} \phi_{R}-\left(\mathbf{A}_{R}\right)_{\xi} \Phi_{R}\right]\right]^{T}+\right. \\
& \frac{1}{2}\left[\mathbf{V}^{T} P_{R}\left[\left(\mathbf{B}_{R}\left(D_{\eta}\right)_{R} \phi_{R}+\left(D_{\eta}\right)_{R} \mathbf{B}_{R} \phi_{R}-\left(\mathbf{B}_{R}\right)_{\eta} \Phi_{R}\right]\right]^{T}=\right. \\
& -\phi_{R}^{T}\left(P_{\tau} \otimes E_{0} \otimes P_{\eta} \otimes I\right) \mathbf{A}_{R} \mathbf{V}-\phi_{R}^{T}\left(P_{\tau} \otimes E_{0} \otimes P_{\eta} \otimes I\right) \Sigma_{R}(\mathbf{V}-\mathbf{U}) .
\end{aligned}
$$

Next, we add and subtract the terms $\left[\mathbf{U}^{T} P_{L}\left(\left(\mathbf{J}_{L}\right)_{\tau}+\left(\mathbf{A}_{L}\right) \xi+\left(\mathbf{B}_{L}\right)_{\eta}\right) \phi_{L}\right]^{T}$ and $\left[\mathbf{V}^{T} P_{R}\left(\left(\mathbf{J}_{R}\right)_{\tau}+\right.\right.$ $\left.\left.\left(\mathbf{A}_{R}\right) \xi+\left(\mathbf{B}_{R}\right)_{\eta}\right) \phi_{R}\right]^{T}$ to the first and second equations in (66), respectively, and 
obtain

$$
\begin{gathered}
\frac{1}{2}\left[\mathbf { U } ^ { T } P _ { L } \left(\left[\mathbf{J}_{L}\left(D_{\tau}\right)_{L}+\left(D_{\tau}\right)_{L} \mathbf{J}_{L}+\left(\mathbf{J}_{L}\right)_{\tau}\right] \phi_{L}+\left[\left(\mathbf{A}_{L}\left(D_{\xi}\right)_{L}+\left(D_{\xi}\right)_{L} \mathbf{A}_{L}+\left(\mathbf{A}_{L}\right)_{\xi}\right] \phi_{L}+\right.\right.\right. \\
\left.\left.\left[\mathbf{B}_{L}\left(D_{\eta}\right)_{L}+\left(D_{\eta}\right)_{L} \mathbf{B}_{L}+\left(\mathbf{B}_{L}\right)_{\eta}\right] \phi_{L}\right)\right]^{T}=\phi_{L}^{T}\left(P_{\tau} \otimes E_{1} \otimes P_{\eta} \otimes I\right) \mathbf{A}_{L} \mathbf{U} \\
-\phi_{L}^{T}\left(P_{\tau} \otimes E_{1} \otimes P_{\eta} \otimes I\right) \Sigma_{L}(\mathbf{U}-\mathbf{V})-\left[\mathbf{U}^{T} P_{L}\left[\left(\mathbf{J}_{L}\right)_{\tau}+\left(\mathbf{A}_{L}\right)_{\xi}+\left(\mathbf{B}_{L}\right)_{\eta}\right] \phi_{L}\right]^{T}, \\
\frac{1}{2}\left[\mathbf { V } ^ { T } P _ { R } \left(\left[\mathbf{J}_{R}\left(D_{\tau}\right)_{R}+\left(D_{\tau}\right)_{R} \mathbf{J}_{R}+\left(\mathbf{J}_{R}\right)_{\tau}\right] \phi_{R}+\left[\left(\mathbf{A}_{R}\left(D_{\xi}\right)_{R}+\left(D_{\xi}\right)_{R} \mathbf{A}_{R}+\left(\mathbf{A}_{R}\right)_{\xi}\right] \phi_{R}+\right.\right.\right. \\
\left.\left.\left[\mathbf{B}_{R}\left(D_{\eta}\right)_{R}+\left(D_{\eta}\right)_{R} \mathbf{B}_{R}+\left(\mathbf{B}_{R}\right)_{\eta}\right] \phi_{R}\right)\right]^{T}=-\phi_{R}^{T}\left(P_{\tau} \otimes E_{0} \otimes P_{\eta} \otimes I\right) \mathbf{A}_{R} \mathbf{V} \\
-\phi_{R}^{T}\left(P_{\tau} \otimes E_{0} \otimes P_{\eta} \otimes I\right) \Sigma_{R}(\mathbf{V}-\mathbf{U})+\left[\mathbf{V}^{T} P_{R}\left[\left(\mathbf{J}_{R}\right)_{\tau}+\left(\mathbf{A}_{R}\right)_{\xi}+\left(\mathbf{B}_{R}\right)_{\eta}\right] \phi_{R}\right]^{T} .
\end{gathered}
$$

By applying the NGCL to the right hand side of both equations in (67), and adding the two results, we get (29). 


\section{References}

[1] S. Nikkar and J. Nordström, Fully discrete energy stable high order finite difference methods for hyperbolic problems in deforming domains, Journal of Computational Physics, 291:82-98 (2015).

[2] M. Svärd and J. Nordström, Review of summation-by-parts schemes for initial-boundary-value problems, Journal of Computational Physics, 268:17-38 (2014).

[3] J. Nordström and J. Gong and E. van der Weide and M. Svärd, A stable and conservative high order high order multi-block method for the compressible Navier-Stokes equations, Journal of Computational Physics, 228(24):90209035 (2009).

[4] B. Strand, Summation by parts for finite difference approximations of $d / d x$, Journal of Computational Physics, 110(1):47-67 (1994).

[5] J. Gong and J. Nordström, Interface procedures for finite difference approximations of the advection-diffusion equation, Journal of Computational and Applied Mathematics, 236(5):602-620 (2011).

[6] K. Mattsson and M. H. Carpenter, Stable and accurate interpolation operators for high-order multi-block finite-difference methods, SIAM Journal of Scientific Computing, 32(4):2298-2320 (2010).

[7] J. Nordström and T. Lundquist, Summation-by-parts in time, Journal of Computational Physics, 251:487-499 (2013).

[8] T. Lundquist and J. Nordström, The SBP-SAT technique for initial value problems, Journal of Computational Physics, 270:86-104 (2014).

[9] M. Svärd and J. Nordström, On the order of accuracy for difference approximations of initial boundary value problems, Journal of Computational Physics, 218(1):333-352 (2006).

[10] M. H. Carpenter and J. Nordström and D. Gottleib, A stable and conservative interface treatment of arbitrary spatial accuracy, Journal of Computational Physics, 148(2):341-365 (1999). 
[11] M. H. Carpenter and J. Nordström and D. Gottleib, Revising and extending interface penalties for multi-domain summation-by-parts operators, Journal of Scientific Computing, 45(1-3):118-150 (2010).

[12] C. Farhat and P. Geuzaine and C. Grandmont, The discrete geometric conservation law and the nonlinear stability of ALE schemes for the solution of flow problems on moving grids, Journal of Computational Physics, 174(2):669-694 (2001).

[13] C. F. Van Loan, The ubiquitous Kronecker product, Journal of Computational and Applied Mathematics, 123(1):85-100 (2000).

[14] S. S. Abarbanel and A. E. Chertock, Strict stability of high-order compact implicit finite-difference schemes: the role of boundary conditions for hyperbolic PDEs, I, Journal of Computational Physics, 160(1):42-66 (2000).

[15] S. S. Abarbanel and A. E. Chertock and A. Yefet, Strict stability of highorder compact implicit finite-difference schemes: the role of boundary conditions for hyperbolic PDEs, II, Journal of Computational Physics, 160:6787 (2000).

[16] M. H. Carpenter and D. Gottlieb, Spectral methods on arbitrary grids, Journal of Computational Physics, 129(1):74-86 (1996).

[17] D. C. Del Rey Fernández and P. D. Boom and D. W. Zingg, A generalized framework for nodal first derivative summation-by-parts operators, Journal of Computational Physics, 266:214-239 (2014).

[18] J. Nordström, Conservative finite difference formulations, variable coefficients, energy estimates and artificial dissipation, Journal of Scientific Computing, 29(3):375-404 (2006).

[19] E. Turkel, Symmetrization of the fluid dynamics matrices with applications, Mathematics of Computation, 27(124):729-736 (1973).

[20] B. Gustafsson, The convergence rate for difference approximation to mixed initial boundary value problems, Mathematics of Computation, 29(130):396-406 (1975)

[21] B. Gustafsson, The convergence rate for difference approximation to general mixed initial-boundary value problems, SIAM Journal on Numerical Analysis, 18(2):179-190 (1981). 
[22] C. W. Hirt and A. A. Amsden and J. L. Cook, An arbitrary LagrangianEulerian computing method for all flow speeds, Journal of Computational Physics, 14(3):227-253 (1974).

[23] Y. Abe and N. Iizuka and T. Nonomura and K. Fujii, Conservative metric evaluation for high-order finite-difference schemes with the GCL identities on moving and deforming grids, Journal of Computational Physics, 232(1):14-21 (2013).

[24] T. Lundquist and J. Nordström, On the suboptimal accuracy of summationby-parts schemes with non-conforming block interfaces, LiTH-MAT-R2015/16-SE.

[25] D. McCarthy and B. D. McKay, Transposable and symmetrizable matrices, Journal of Australian Mathematical Society, 29(4):469-474 (1980).

[26] B. Sjögreen and H. C. Yee, Variable high order multiblock overlapping grid methods for mixed steady and unsteady multiscale viscous flows, Соттиnications in Computational Physics, 5:730-744 (2009).

[27] X. Huan and J. E. Hicken and D. Zingg, Interface and boundary scheme for high-order methods, The 39th AIAA Computational Fluid Dynamics Conference, AIAA Paper, No. 2009-3658 (2009).

[28] A. Nissen and G. Kreiss and M. Gerritsen, Stability at nonconforming grid interfaces for a high order discretization of the Schrödinger equation, Journal of Scientific Computing, 53(3):528-551 (2012).

[29] A. Nissen and G. Kreiss and M. Gerritsen, High order stable finite difference methods for the Schrödinger equation, Journal of Scientific Computing, 55(1):173-199 (2013).

[30] P. D. Thomas and C. K. Lombard, Geometric conservation law and its application to flow computations on moving grids, AIAA Journal, 17(10):10301037 (1979).

[31] J. Nordström, Error bounded schemes for time-dependent hyperbolic problems, SIAM Journal of Scientific Computing, 30:46-59 (2007).

[32] P. Lax, B. Wendroff, Systems of conservation laws, Pure and Applied Mathematics, 13:217-237 (1960). 